\title{
BORNOLOGICAL STRUCTURES ON MANY-VALUED SETS
}

\author{
Alexander Šostak And Ingrīda Uluane
}

Dedicated to the memory of Professor Sibe Mardešić

\begin{abstract}
We introduce an approach to the concept of bornology in the framework of many-valued mathematical structures and develop the basics of the theory of many-valued bornological spaces and initiate the study of the category of many-valued bornological spaces and appropriately defined bounded "mappings" of such spaces. A scheme for constructing many-valued bornologies with prescribed properties is worked out. In particular, this scheme allows to extend an ordinary bornology of a metric space to a many-valued bornology on it.
\end{abstract}

In the academic year 1978/79 I was lucky to get a post-doc research position at the University of Zagreb and to spend this time working in collaboration with professor Sibe Mardešić in shape theory. It was a real pleasure to work together and to communicate generally with this outstanding mathematician, talented teacher and a very kind and nice man. Unfortunately, at present I am quite far from shape theory. Therefore the paper I would like to dedicate to Sibe Mardešić, written together with my former PhD student Ingrīda Uljane, is in the field of so called many-valued mathematical structures - the area of my main mathematical interests at present.

\section{InTRODUCTION AND MOTIVATION}

1.1. Bornologies and bornological spaces.

In order to apply the conception of boundedness to the case of a general topological space, Hu S.T. introduced the notions of a bornology and a bornological space [21]. A bornology on a set $X$ is a family $\mathbb{B} \subseteq 2^{X}$ such that

(1B) $\bigcup\{U \mid U \in \mathbb{B}\}=X$;

(2B) if $U \subseteq V$ and $V \in \mathbb{B}$ then $U \in \mathbb{B}$;

(3B) if $U, V \in \mathbb{B}$ then $U \cup V \in \mathbb{B}$.

2010 Mathematics Subject Classification. 54A40.

Key words and phrases. Bornology, quantale, many-valued mathematical structures, many-valued bornology, fuzzy set. 
The pair $(X, \mathbb{B})$ is called a bornological space and the sets belonging to $\mathbb{B}$ are viewed as bounded in this space. Given bornological spaces $\left(X, \mathbb{B}_{X}\right)$ and $\left(Y, \mathbb{B}_{Y}\right)$, a mapping $f:\left(X, \mathbb{B}_{X}\right) \rightarrow\left(Y, \mathbb{B}_{Y}\right)$ is called bounded if $f(A) \in \mathbb{B}_{Y}$ for every $A \in \mathbb{B}_{X}$.

In the original definition of bornology instead of axiom (1B) the following axiom

$\left(1^{\prime} \mathrm{B}\right) \forall x \in X \Rightarrow\{x\} \in \mathbb{B}$

was used. It is easy to see that under assumption of axiom (2B) axiom (1B) is equivalent to axiom $\left(1^{\prime} \mathrm{B}\right)$. We use the axiom $(1 \mathrm{~B})$ instead of $\left(1^{\prime} \mathrm{B}\right)$ as more appropriate for the principal merits of this work.

Important examples of bornological spaces $(X, \mathbb{B})$ are:

- a metric space and the family of its bounded subsets;

- a topological space and and the family of its relatively compact subsets;

- a uniform space and and the family of its totally bounded subsets.

At present the theory of bornological spaces is developed in various directions. Most of the research involving bornologies is done in the context of topological linear spaces, see e.g. [36], [14], and in topological algebras, that is in cases when the underlying set, besides topology, is endowed with a certain algebraic structure. However, a notable research work, especially in recent time, is being done in the field of bornologies without referring to the algebraic structure of the underlying set. Specifically, much interest is in the research of bornological universes that is triples $(X, \mathcal{T}, \mathbb{B})$, where $\mathcal{T}$ is a topology and $\mathbb{B}$ is a bornology on a set $X$. General bornological spaces play a key role in recent research of convergence structures on hyperspaces [2], [3], [26], in optimization theory [5] and in the study of topologies on function spaces [4], [29].

The principal aim of this work is to make a contribution to the creation of bornological theories in the context of many-valued mathematical structures, or, as this area of mathematics is often referred to, in the field of "Fuzzy Mathematics". At present there are only few works dealing with this problem, see [1], [42], [30]. It is quite different from the situation in "Fuzzy Topology", which is a very well developed area of theoretical mathematics with many fundamental concepts, constructions and results, and which has important applications to other fields of mathematics, see e.g. [27], [19].

1.2. Bornologies on L-powersets.

In [1] a bornological structure on the family $L^{X}$ of $L$-fuzzy subsets of a set $X$ was defined. In the sequel referring to such structures we call them $L$ bornologies. Namely, given a frame, that is a completely distributive lattice, and a set $X$, an $L$-bornology on the set $X$ is a subset $\mathbb{B}$ of the family $L^{X}$ of $L$-fuzzy subsets of $X$ such that

(1LB) $\bigvee\{B \mid \mathcal{B} \in \mathbb{B}\}=1_{L^{X}}$ 
(2LB) if $A \leq B$ and $B \in \mathbb{B}$ then $A \in \mathbb{B}$;

(3LB) if $A, B \in \mathbb{B}$ then $A \vee B \in \mathbb{B}$.

The pair $(X, \mathbb{B})$ is called an L-bornological space.

Such approach to the problem of "fuzzification" of bornology is the bornological analogue of Chang-Goguen's definition of a fuzzy topology [7], [13].

In [1] also a stronger version of the axiom (1LB), namely

$\left(1^{\prime} \mathrm{LB}\right) \forall x \in X \Rightarrow \chi_{\{x\}} \in \mathbb{B}$, where $\chi_{\{x\}}$ is the characteristic function of the one-element set $\{x\}$,

was considered. The corresponding structure $\mathbb{B}$ will be called a strong $L$ bornology. In case when $1_{L}$ is an isolated element of the lattice $L$ the concepts of $L$-bornology and strong $L$-bornology are equivalent.

1.3. M-valued bornologies on power-sets.

An alternative approach to the problem of fuzzification of the concept of bornology was presented in [41], and further developed in [43] and [42]. In the sequel we will refer to this approach as an $M$-valued bornology on a set $X$. Namely, an $M$-valued bornology on a set $X$ where $M$ is a complete lattice, is defined as a mapping $\mathcal{B}: 2^{X} \rightarrow M$ such that

(1MB) $\forall x \in X \Rightarrow \mathcal{B}(\{x\})=1_{M}$ where $1_{M}$ is the top element of $M$;

(2MB) if $A \subseteq B$ then $\mathcal{B}(A) \geq \mathcal{B}(B)$;

(3MB) for every $A, B \subseteq X$ it holds $\mathcal{B}(A \cup B) \geq \mathcal{B}(A) \wedge \mathcal{B}(B)$.

The pair $(X, \mathcal{B})$ is called an $M$-valued bornological space.

Such approach to the problem of "fuzzification" of bornology is the bornological analogue of Höhle-Ying's approach to a fuzzy topology [15], [45].

In this paper we consider the concept of an $M$-valued bornology on the $L$-powerset of a set $X$ defined as a mapping $\mathcal{B}: L^{X} \rightarrow M$ satisfying conditions analogous to the properties (1MB) - (3MB). Thus this approach, on one hand is the "roof" for the approaches presented in subsections 1.2 and 1.3, and on the other hand is the bornological analogue of the approach to fuzzification of topologies presented in [25], [37], [38]. However, having an intention to develop our approach in the sufficiently general framework, we will assume that, instead of an ordinary set $X$, we deal with a many-valued set, that is a set endowed with some many-valued equality. As an additional incentive to develop our approach in the general framework of many-valued sets instead of ordinary sets, we consider the role of many-valued sets in the theory of fuzzy topologies: just in this framework many interesting results and examples are obtained, in particular applications related to other fields of theoretical mathematics, see e.g. [19]. 
The paper has the following structure. After recalling in Section 2 basic concepts needed throughout the paper, we introduce the concept of an $M$ valued bornology on the $L$-powerset of an $L$-valued set, which will be referred to as an $L M$-valued bornology for short, and discuss some properties of $L M$ valued bornologies in Section 3. Our next aim is to develop, what can be called, the categorical basics of the theory of $L M$-valued bornological spaces. This problem is being considered in Section 5. However, before turning to this problem first we have to outline the class of potential morphisms for this category. $L M$-valued bornologies are defined on the basis of $L$-valued sets and just fuzzy functions are used as "natural" morphisms between $L$-valued sets. Therefore, before introducing the category of $L M$-bornological spaces and bounded fuzzy functions in Section 5, we present a brief introduction in the theory of fuzzy functions in Section 4. In Section 7 we consider a general scheme for construction of $L M$-valued bornologies on metric spaces. In the last, Conclusion Section 8, we discuss some directions for the further research of $L M$-valued bornologies.

\section{Prerequisites: The context of the Work}

2.1. Lattices and quantales.

In this work two objects, $\mathbf{L}$ and $\mathbf{M}$, will play the fundamental role.

By $\mathbf{L}=\left(L, \leq_{L}, \wedge_{L}, \vee_{L}, *\right)$ we will denote an integral commutative quantale. Following e.g. [19], see also [35], by an integral commutative quantale ${ }^{1}$ we call a tuple $\mathbf{L}=\left(L, \leq_{L}, \wedge_{L}, \vee_{L}, *\right)$ where $\left(L, \leq_{L}, \wedge_{L}, \vee_{L}\right)$ is a complete lattice that is a lattice in which arbitrary suprema (joins) and infima (meets) exist, in particular, the top $1_{L}$ and the bottom $0_{L}$ elements in $L$ exist, and the binary operation $*: L \times L \rightarrow L$ satisfies conditions:

$(0 \mathrm{cl}) *$ is monotone: $\alpha \leq_{L} \beta \Longrightarrow \alpha * \gamma \leq_{L} \beta * \gamma$ for all $\alpha, \beta, \gamma \in L$;

$(1 \mathrm{cl}) *$ is commutative: $\alpha * \beta=\beta * \alpha$ for all $\alpha, \beta \in L$;

$(2 \mathrm{cl}) *$ is associative: $(\alpha * \beta) * \gamma=\alpha *(\beta * \gamma)$ for all $\alpha, \beta, \gamma \in L$;

$(3 \mathrm{cl}) *$ distributes over arbitrary joins: $\alpha *\left(\bigvee_{i \in I} \beta_{i}\right)=\bigvee_{i \in I}\left(\alpha * \beta_{i}\right)$ for all $\alpha \in L$, for all $\left\{\beta_{i} \mid i \in I\right\} \subseteq L$,

(4cl) $\alpha * 1_{L}=\alpha$ for all $\alpha \in L$.

It is known and easy to prove that $\alpha * 0_{L}=0_{L}$ for every $\alpha \in L$.

In an integral commutative quantale a further binary operation $\mapsto$, residuation, is defined:

$$
\alpha \mapsto \beta=\bigvee\{\lambda \in L \mid \lambda * \alpha \leq \beta\} \forall \alpha, \beta \in L .
$$

Residuation is connected with operation $*$ by Galois connection, see [11]:

$$
\alpha * \beta \leq \gamma \Longleftrightarrow \alpha \leq(\beta \mapsto \gamma)
$$

\footnotetext{
${ }^{1}$ Integral commutative quantales are known also as integral commutative complete lattice monoids, see e.g. [16]
} 
In the sequel $L$ will always denote an arbitrary integral commutative quantale whose underlying lattice is a frame that is

$$
\alpha \wedge_{L}\left(\bigvee_{i} \beta_{i}\right)=\bigvee_{i}\left(\alpha \wedge_{L} \beta_{i}\right) \quad \forall \alpha \in L, \forall\left\{\beta_{i}: i \in I\right\} \subseteq L
$$

By $\mathbf{M}$ we denote a complete completely distributive lattice $\mathbf{M}=\left(M, \leq_{M}\right.$, $\wedge_{M}, \vee_{M}$ ) whose bottom and top elements are denoted by $0_{M}$ and $1_{M}$ respectively. Actually we will use not the original definition of complete distributivity, see e.g [11, Definition I-2-8], but its characterization given by G.N. Raney [33]. Namely, given a complete lattice $M$ and $\beta, \alpha \in M$ following [33], see also [11, Excercise IV-3-31], we introduce the so called "wedge below" relation $\triangleleft$ on $M$ as follows:

$$
\beta \triangleleft \alpha \Longleftrightarrow(\text { if } K \subseteq M \text { and } \alpha \leq \bigvee K \text { then } \exists \gamma \in K, \beta \leq \gamma) .
$$

As shown by G.N. Raney [33] a lattice $M$ is completely distributive if and only if relation $\triangleleft$ has the approximation property, that is

$$
\alpha=\bigvee\{\beta \in M \mid \beta \triangleleft \alpha\} \text { for every } \alpha \in M .
$$

Moreover, relation $\triangleleft$ has the following nice properties (see $[11,33]$ ) used in the sequel:

$(\triangleleft 1) \beta \triangleleft \alpha$ implies $\beta \leq \alpha$;

$(\triangleleft 2) \gamma \leq \beta \triangleleft \alpha \leq \delta$ implies $\gamma \triangleleft \delta$;

$(\triangleleft 3) \beta \triangleleft \alpha$ implies that there exists $\gamma \in L$ such that $\beta \triangleleft \gamma \triangleleft \alpha$.

We denote $M^{\circ}=\left\{\alpha \in M \mid \alpha \triangleleft 1_{M}\right\}$. Note that $1_{M} \in M^{\circ}$ if and only if the top element $1_{M}$ is not isolated in $M$.

In the sequel, when dealing with lattice $L$ and $M$ we usually omit subscripts $\leq_{L}, \leq_{M}$, etc, when it will not make misunderstanding.

2.2. Fuzzy sets. [46], [12].

Recall that an $(L)$-fuzzy subset of a set $X$, where $L$ is a complete lattice, is a mapping $A: X \rightarrow L$. Given a family $\left\{A_{i} \mid i \in I\right\}$ its union $\bigvee_{i} A_{i}: X \rightarrow L$ and intersection $\bigwedge_{i} A_{i}: X \rightarrow L$ are defined respectively by

$$
\left(\bigvee_{i} A_{i}\right)(x)=\sup _{i \in I} A_{i}(x),\left(\bigwedge_{i} A_{i}\right)(x)=\inf _{i \in I} A_{i}(x)
$$

2.3. L-relations, L-valued equalities and L-valued sets.

Given sets $X, Y$ and a quantale $L$, by an $L$-relation between $X$ and $Y$ we call a mapping $R: X \times Y \rightarrow L$. In case $X=Y$, an $L$-relation $E: X \times X \rightarrow L$ is called an L-valued equivalence if it is

(1E) reflexive, that is $E(x, x)=1_{L}$ for every $x \in X$;

(2E) symmetric, that is $E(x, y)=E(y, x)$ for all $x, y \in X$;

(3E) transitive, that is $E(x, y) * E(y, z) \leq E(x, z)$ for all $x, y, z \in X$. 
An $L$-valued equivalence $E: X \times X \rightarrow L$ is called an $L$-valued equality if it is separated, that is $E(x, y)=1_{L} \Rightarrow x=y$. A pair $(X, E)$, where $E: X \times X \rightarrow L$ is an $L$-valued equality on $X$, is called an $L$-valued, or a many-valued, set. ${ }^{2}$

When dealing with fuzzy subsets of $L$-valued sets, the property of extensionality plays an important role. This property was considered by many authors, see e.g. U. Höhle [16], [17] and F. Klawon [23]:

A fuzzy set $A$ in an $L$-valued set $(X, E)$ is called extensional if

$$
A(x) * E\left(x, x^{\prime}\right) \leq A\left(x^{\prime}\right) \forall x, x^{\prime} \in X .
$$

The smallest extensional fuzzy set $e(A)$ in $(X, E)$ that is larger than or equal to $A$ (i.e. $A \leq e(A))$ is called the extensional hull of $A$. Explicitly the extensional hull of $A$ can be defined as follows (see e.g. [16], [17], [23]):

$$
e(A)(x)=\bigvee_{x^{\prime} \in X}\left(E\left(x, x^{\prime}\right) * A\left(x^{\prime}\right)\right) .
$$

In particular, identifying an element $x_{0}$ with the characteristic function $\chi_{\left\{x_{0}\right\}}$ of the one-element set $\left\{x_{0}\right\}$, we get the extensional hull of the point $x_{0}$, sometimes called a fuzzy singleton:

$$
e\left(x_{0}\right)(x)=E\left(x_{0}, x\right) .
$$

\section{LM-VALUED BORNOLOGIES}

3.1. M-valued bornologies on L-powersets of L-valued sets: Basic definitions.

Let $\mathbf{L}=\left(L, \leq_{L}, \wedge_{L}, \vee_{L}, *\right)$ be an integral commutative quantale, $\mathbf{M}=$ $\left(M, \leq_{M}, \wedge_{M}, \vee_{M}\right)$ be a completely distributive lattice and $E: X \times X \rightarrow L$ be an $L$-valued equality on the set $X$.

Definition 3.1. By an $M$-valued bornology on the $L$-powerset of an $L$ valued set $(X, E)$, or an LM-valued bornology on $(X, E)$ for short, we call a mapping $\mathcal{B}: L^{X} \rightarrow M$ satisfying the following conditions:

(1LMB) $\forall \alpha \in M^{\circ} \exists \mathcal{U} \subseteq L^{X}$ such that $\bigvee \mathcal{U}=1_{L^{X}}$ and $\mathcal{B}(U) \geq \alpha \forall U \in \mathcal{U}$;

(2LMB) $\quad A \leq B \Rightarrow \mathcal{B}(A) \geq \mathcal{B}(B) \forall A, B \in L^{X}$;

$(3 \mathrm{LMB}) \quad \mathcal{B}(\bar{A} \vee B) \geq \mathcal{B}(A) \wedge \mathcal{B}(B) \forall A, B \in L^{X}$;

(4LMB) $\quad \mathcal{B}(e(A))=\mathcal{B}(A) \forall A \in L^{X}$.

The triple $(X, E, \mathcal{B})$, where $(X, E)$ is an L-valued set and $\mathcal{B}: L^{X} \rightarrow M$, is an $L M$-valued bornology on it is called an LM-valued bornological space.

REmark 3.2. 1. The value $\mathcal{B}(A)$ is interpreted as the degree of boundedness of a fuzzy set $A \in L^{X}$ in the $L M$-valued bornology $\mathcal{B}$.

2. The axioms (2LMB) and (3LMB) together are equivalent to the following axiom

${ }^{2}$ The concepts called here an $L$-relation an $L$-valued equivalence and $L$-valued equality under different names and with different degrees of generality appear in many papers, see e.g. [44], [47], [6], etc. 
$\left(3^{\wedge} \mathrm{LMB}\right) \mathcal{B}(A \vee B)=\mathcal{B}(A) \wedge \mathcal{B}(B)$ for all $A, B \in L^{X}$.

However for certain reasons, we prefer to split axiom $\left(3^{\wedge} \mathrm{LMB}\right)$ in the definition of an $L M$-valued bornology into two separate axioms.

3. In case when the relation $E$ is the ordinary relation $=$ on a set $X$, that is $E(x, y)=1$ if and only if $x=y$, condition (4LMB) holds trivially. In particular, if besides $M=2$ is the two-element lattice, then our definition of an $2 M$-valued bornology reduces to the definition of an $L$-bornology from Subsection 1.2. On the other hand, if $L=2$ and $E$ is still the ordinary equality relation, then we come to the definition of an $M$-valued bornology from Subsection 1.3. Finally, in case if the both lattices are two-point, that is $L=M=2$, we return to the original definition of a bornology [21], see Subsection 1.1.

One can prefer to consider the following stronger version of the first axiom: $\left(1^{\prime} \mathrm{LMB}\right) \bigvee\left\{U \mid U \in L^{X}, \mathcal{B}(U)=1_{M}\right\}=1_{L^{X}}$.

We call the mapping $\mathcal{B}: L^{X} \rightarrow M$ satisfying conditions (1'LMB), (2LMB), (3LMB) and (4LMB) a strong LM-valued bornology on $(X, E)$. Obviously, in case $1_{M}$ is an isolated element in $M$, the concepts of an $L M$-valued bornology and a strong $L M$-valued bornology are equivalent.

3.2. Lattice of $L M$-valued bornologies.

We introduce a partial order relation $\preceq$ on the set $\mathfrak{B}(L, M, X, E)$ of all $L M$-valued bornologies on an $L$-valued set $(X, E)$ by setting for $\mathcal{B}_{1}, \mathcal{B}_{2} \in$ $\mathfrak{B}(L, M, X, E)$ :

$$
\mathcal{B}_{1} \preceq \mathcal{B}_{2} \Longleftrightarrow \mathcal{B}_{1}(A) \geq \mathcal{B}_{2}(A) \forall A \in L^{X},
$$

and say in this case that $\mathcal{B}_{1}$ is coarser than $\mathcal{B}_{2}$, and $\mathcal{B}_{2}$ is finer than $\mathcal{B}_{1}$. We show that the partially ordered set $\mathfrak{B}(L, M, X, E, \preceq)$ is a complete lattice.

By setting $\mathcal{B}_{\perp}(A)=1_{M}$ for all $A \in L^{X}$ we get the coarsest $L M$-valued bornology on an $L$-valued set $(X, E)$, that is $\mathcal{B}_{\perp}$ is the bottom element in $\mathfrak{B}(L, M, X, E, \preceq)$. To define the finest element $\mathcal{B}_{\top}$ in $\mathfrak{B}(L, M, X, E, \preceq)$, we introduce the following notation. Let $S \subseteq X$ and $\lambda \in L$. Then we define a fuzzy set $S^{\lambda}: X \rightarrow L$ by setting:

$$
S^{\lambda}(x)=\left\{\begin{aligned}
\lambda & \text { if } x \in S \\
0_{L} & \text { otherwise }
\end{aligned}\right.
$$

Now by setting:

$$
\mathcal{B}_{\top}(A)= \begin{cases}1_{M} & \text { if } \exists S \subseteq X,|S|<\aleph_{0}, \exists \lambda \text { such that } A \leq e\left(S^{\lambda}\right) ; \\ 0_{M} & \text { otherwise. }\end{cases}
$$

we obtain the finest $L M$-valued bornology on an $L$-valued set $(X, E)$, that is the top element in $\mathfrak{B}(L, M, X, E, \preceq)$.

Further, given a family $\left\{\mathcal{B}_{i}: L^{X} \rightarrow M \mid i \in I\right\}$ of $L M$-valued bornologies, we define $\curlyvee_{i \in I} \mathcal{B}_{i}=: \mathcal{B}_{0}: L^{X} \rightarrow M$ by setting $\mathcal{B}_{0}(A)=\bigwedge_{i \in I} \mathcal{B}_{i}(A)$. 
One can easily see that the mapping $\curlyvee_{i \in I} \mathcal{B}_{i}: L^{X} \rightarrow M$ thus obtained is an $L M$-valued bornology on $(X, E)$. Indeed, the validity of properties (1LMB), (2LMB) and (4LMB) for $\curlyvee_{i \in I} \mathcal{B}_{i}: L^{X} \rightarrow M$ obviously follows from the corresponding properties of the $L M$-valued bornologies $\mathcal{B}_{i}$. We establish property (3LMB) as follows. Given $A, B \in L^{X}$ we have:

$$
\mathcal{B}_{0}(A \vee B)=\bigwedge_{i \in I} \mathcal{B}_{i}(A \vee B) \geq \bigwedge_{i}\left(\mathcal{B}_{i}(A) \wedge \mathcal{B}_{i}(B)\right)=\mathcal{B}_{0}(A) \wedge \mathcal{B}_{0}(B)
$$

Besides, from the construction it is clear that $\curlyvee_{i \in I} \mathcal{B}_{i}$ is the lowest upper bound of the family $\left\{\mathcal{B}_{i} \mid i \in I\right\}$ in $\mathfrak{B}(L, M, X, E, \preceq)$.

This already guarantees that the family $\mathfrak{B}(L, M, X, E, \preceq, \curlyvee)$ of $L M$ valued bornologies is a complete join semi-lattice. Notice however, that the point-wise supremum $\bigvee_{i}\left(\mathcal{B}_{i}(A)\right)$ of the family $\left\{\mathcal{B}_{i}: L^{X} \rightarrow M \mid i \in I\right\}$ need not be an $L M$-valued bornology (axiom (3LMB) may be violated). Therefore the infimum $\curlywedge$ of the family $\left\{\mathcal{B}_{i}: L^{X} \rightarrow M \mid i \in I\right\}$ in the partially ordered set $\mathfrak{B}(L, M, X, E, \preceq, \curlyvee)$ is defined by

$$
\curlywedge_{i \in I} \mathcal{B}_{i}=\curlyvee\left\{\mathcal{B} \in \mathfrak{B}(L, M, X, E, \preceq, \curlyvee) \mid \mathcal{B} \leq \bigwedge_{i}\left(\mathcal{B}_{i}\right)\right\} .
$$

REMARK 3.3. Let $\mathfrak{B}^{s}(L, M, X, E)$ denote the family of all strong $L M$ valued bornologies on $(X, E)$. It is clear that $\mathfrak{B}^{s}(L, M, X, E)$ is a complete sublattice of $\mathfrak{B}(L, M, X, E, \preceq, \curlyvee)$. Its upper element $\mathcal{B}_{T}$ can be defined by

$$
\mathcal{B}_{\top}(A)= \begin{cases}1_{M} & \text { if } \exists S \subseteq X,|S|<\aleph_{0} \text { such that } A \leq e\left(1_{S}\right) ; \\ 0_{M} & \text { otherwise. }\end{cases}
$$

where $1_{S}$ is the characteristic function of $S$.

3.3. Decomposition of an L-valued bornology into a family of level bornologies.

Given an $M$-valued bornology $\mathcal{B}: L^{X} \rightarrow M$ on an $L$-valued set and $\alpha \in M$, we define a family of $L$-subsets of $X$ :

$$
\mathcal{B}_{\alpha}=\left\{A \in L^{X} \mid \mathcal{B}(A) \geq \alpha\right\} .
$$

One can easily see that the family $\mathcal{B}_{\alpha}$ is an $L$-bornology (Subsection 1.2 ) on the set $X$. Further, taking into account that in a completely distributive lattice every element is the supremum of a family of wedge-below elements, one can easily notice that the family of $\alpha$-levels $\left\{\mathcal{B}_{\alpha} \mid \alpha \in M\right\}$ of an $M$-valued bornology is lower semi-continuous, in the sense that

$$
\mathcal{B}_{\alpha}=\bigcap\left\{\mathcal{B}_{\beta} \mid \beta \triangleleft \alpha, \beta \in M\right\} \text { for every } \alpha \in M,
$$

in particular, $\mathcal{B}_{0_{M}}=L^{X}$ since $0_{M} \triangleleft 0_{M}$. In the special case when $M=[0,1]$ is the unit interval with the "less" ordering $<$, we have $\mathcal{B}_{\alpha}=\bigcap\left\{\mathcal{B}_{\beta} \mid \beta<\right.$ $\alpha$ \} for every $\alpha>0_{M}$. 
Thus in case of a completely distributive lattice every $M$-valued bornology $\mathcal{B}$ can be characterized by its lower semi-continuous decomposition into level $L$-bornologies: $\left\{\mathcal{B}_{\alpha}=\bigvee_{\beta \triangleleft \alpha} \mathcal{B}_{\beta} \mid \alpha \in M\right\}$.

3.4. Construction of an $M$-valued bornology from a family of L-bornologies on an L-valued set.

As opposite to the previous subsection here we present a construction of an $L M$-valued bornology from an indexed family of $L$-bornologies.

Let $\mathbf{M}=(M, \leq, \wedge, \vee)$ be a complete completely distributive lattice, and let $K$ be a subset of $M$, such that

$\left(\mathrm{K}_{\triangleleft}\right) K$ is $\triangleleft$-approximative, that is $\lambda=\sup \{\alpha \in K \mid \alpha \triangleleft \lambda\}$ for each $\lambda \in M$.

From $\left(\mathrm{K}_{\triangleleft}\right)$ and complete distributivity of $M$ it follows that $K$ satisfies the $\triangleleft$-interpolation property, that is

$$
\alpha \triangleleft \beta, \alpha, \beta \in M \Rightarrow \exists \gamma \in K, \alpha \triangleleft \gamma \triangleleft \beta,
$$

In particular, one can take $K=M$ or $K=\mathrm{CP}(M)$, where $\mathrm{CP}(M)$ is the set of all co-primes of the lattice $M$. (Note that $0_{M}$ need not belong to the family $K$ since $0_{M}$ is the supremum of the empty family.) Further, let a non-increasing family of $L$-bornologies on an $L$-valued set $X$ such that be given:

$$
\left\{\mathcal{C}_{\alpha} \mid \alpha \in K\right\} \quad \text { and } \quad \alpha \leq \beta \Longrightarrow \mathcal{C}_{\alpha} \supseteq \mathcal{C}_{\beta},
$$

and assume that $e(A) \in \mathcal{C}_{\alpha}$ whenever $A \in \mathcal{C}_{\alpha}$ Given $A \in L^{X}$ we define $\mathcal{B}(A)=\bigvee\left\{\alpha \in K \mid A \in \mathcal{C}_{\alpha}\right\}$.

Proposition 3.4. The mapping $\mathcal{B}: L^{X} \rightarrow M$ thus defined is an $L M$ valued bornology on the L-valued set $(X, E)$.

Proof. Since every $\mathcal{C}_{\alpha}$ is an $L$-bornology, and hence $\bigvee \mathcal{C}_{\alpha}=1_{L}$, the axiom (1LMB) for $\mathcal{B}$ is ensured by the construction and taking into account the properties of the set $K$.

To show the validity of axiom (2LMB) let $A \leq B ; A, B \in L^{X}$. Then

$$
\mathcal{B}(A)=\bigvee\left\{\alpha \in K \mid A \in \mathcal{C}_{\alpha}\right\} \geq \bigvee\left\{\alpha \in K \mid B \in \mathcal{C}_{\alpha}\right\}=\mathcal{B}(B)
$$

To verify axiom $\left(3^{\prime} \mathrm{LMB}\right)$ let $A, B \in L^{X}$ and assume that

$$
\mathcal{B}(A)=\bigvee\left\{\alpha \in K \mid A \in \mathcal{C}_{\alpha}\right\}:=\lambda, \mathcal{B}(B)=\bigvee\left\{\alpha \in K \mid B \in \mathcal{C}_{\beta}\right\}:=\mu
$$

for some $\lambda, \mu \in M$, but there exists $\nu \triangleleft \lambda \wedge \mu, \nu \in K$ such that $\mathcal{B}(A \vee B) \leq \nu$. Referring to the properties of the set $K$ we can find $\alpha \in K$ such that $\nu \triangleleft \alpha \triangleleft$ $\lambda \wedge \mu$

Then $A \in C_{\alpha}$ and $B \in C_{\alpha}$, and hence $A \vee B \in \mathcal{C}_{\alpha}$. However this means that $\mathcal{B}(A \vee B) \geq \alpha$. The obtained contradiction shows that $\mathcal{B}(A \vee B) \geq \mathcal{B}(A) \wedge \mathcal{B}(B)$.

Finally the validity of (4LMB) follows from the validity of the corresponding property for every $\mathcal{C}_{\alpha}$. 
Proposition 3.5. $\mathcal{B}_{\alpha}=\bigcap\left\{\mathcal{C}_{\beta} \mid \beta \in K, \beta \triangleleft \alpha\right\}$ for every $\alpha \in M$.

Proof. The inclusion $\mathcal{B}_{\alpha} \supseteq \bigcap\left\{\mathcal{C}_{\beta} \mid \beta \in K, \beta \triangleleft \alpha\right\}$ is clear from the construction of the $L M$-valued bornology $\mathcal{B}$. Conversely, if $A \notin \mathcal{B}_{\alpha}$, then there exists $\beta \triangleleft \alpha, \beta \in K$ such that $A \notin \mathcal{C}_{\beta}$. Hence $A \notin \bigcap\left\{\mathcal{C}_{\beta} \mid \beta \in K, \beta \triangleleft \alpha\right\}$.

Given a family of $L$-bornologies $\left\{\mathcal{C}_{\alpha} \mid \alpha \in K\right\}$ on an $L$-valued a set $X$, let the $L M$-valued bornology $\mathcal{B}: L^{X} \rightarrow M$ be defined as above. We define a new family of $L$-bornologies $\left\{\overline{\mathcal{C}}_{\alpha} \mid \alpha \in M\right\}$ by setting $\overline{\mathcal{C}}_{\alpha}:=\bigcap\left\{\mathcal{C}_{\beta} \mid \beta \triangleleft \alpha, \beta \in K\right\}$, where $K \subseteq M$, as before, is assumed to satisfy property $\left(\mathrm{K}_{\triangleleft}\right)$. Further, let the $L M$-valued bornology $\overline{\mathcal{B}}: L^{X} \rightarrow M$ be constructed from this family as above, that is

$$
\overline{\mathcal{B}}(A)=\bigvee\left\{\alpha \in M \mid A \in \overline{\mathcal{C}}_{\alpha}\right\} \text { for every } A \in L^{X} .
$$

Then for every $\alpha \in L$

$$
\overline{\mathcal{B}}_{\alpha}=\bigcap_{\beta \triangleleft \alpha, \beta \in M} \overline{\mathcal{C}}_{\alpha}=\bigcap_{\gamma \triangleleft \beta, \gamma \in K}\left(\bigcap_{\beta \triangleleft \alpha} \mathcal{C}_{\beta}\right)=\bigcap_{\gamma \triangleleft \alpha, \gamma \in K} \mathcal{C}_{\gamma}=\mathcal{B}_{\alpha},
$$

and hence $\overline{\mathcal{B}}=\mathcal{B}$.

\section{FUZZY FUNCTIONS}

In order to define a category with $L M$-valued bornological spaces as objects we have to specify its morphisms. Since our general framework for $L M$-valued bornologies is formed by $L$-valued sets, we take so called fuzzy functions as morphisms for the category of $L M$-bornological spaces. The reason for this choice is that fuzzy functions are well coordinated with $L$-valued equalities and can be interpreted as mappings between $L$-valued sets. In case of ordinary sets, a fuzzy function between them in a natural way can be interpreted as an ordinary function. Therefore, before touching in Section 5 the categorical aspects of the theory of $L M$-valued bornological spaces, in the next section we give a brief introduction into the theory of fuzzy functions.

The concept of a fuzzy function was introduced in [8] and (independently) in [20]. Further fuzzy functions were studied and applied in research of different authors, see [40], [9], [31], [32], etc. Let $\mathbf{L}=(L, \leq, \wedge, \vee, *)$ be a fixed quantale and let $\left(X, E_{X}\right),\left(Y, E_{Y}\right)$ be $L$-valued sets.

Definition 4.1. An L-relation $R: X \times Y \rightarrow L$ is called a fuzzy function from an $L$-valued set $\left(X, E_{X}\right)$ to an L-valued set $\left(Y, E_{Y}\right)$ if

(1ff) $R(x, y) * E_{Y}\left(y, y^{\prime}\right) \leq R\left(x, y^{\prime}\right) \forall x \in X, \forall y, y^{\prime} \in Y$;

(2ff) $E_{X}\left(x, x^{\prime}\right) * R(x, y) \leq R\left(x^{\prime}, y\right) \forall x, x^{\prime} \in X, \forall y \in Y$;

(3ff) $R(x, y) * R\left(x, y^{\prime}\right) \leq E_{Y}\left(y, y^{\prime}\right) \forall x \in X, \forall y, y^{\prime} \in Y$. 
For a fuzzy function from an $L$-valued set $\left(X, E_{X}\right)$ to an $L$-valued set $\left(Y, E_{Y}\right)$ we use notations both $R: X \times Y \rightarrow L$ and $R:\left(X, E_{X}\right) \rightarrow\left(Y, E_{Y}\right)$, giving preference to the one which is more convenient in the context.

REMARK 4.2. Let $E_{X}$ and $E_{Y}$ be ordinary equalities $=_{X}$ and $=_{Y}$ on the sets $X$ and $Y$ respectively. Then an ordinary function $f: X \rightarrow Y$ can be realized as a fuzzy function $R_{f}:\left(X,=_{X}\right) \rightarrow\left(Y,=_{Y}\right)$ that is as an $L$-relation $R_{f}: X \times Y \rightarrow L$ by setting $R_{f}(x, y)= \begin{cases}1_{L} & \text { if } f(x)=y \\ 0_{L} & \text { otherwise. }\end{cases}$

Composition of $L$-relations $R: X \times Y \rightarrow L$ and $S: Y \times Z \rightarrow L$ is the $L$-relation $S \circ R:\left(X, E_{X}\right) \rightarrow\left(Z, E_{Z}\right)$ defined by $(S \circ R)(x, z)=$ $\bigvee_{y \in Y}(R(x, y) * S(y, z))$. In [20], see also [40], it is shown that composition of two fuzzy functions is a fuzzy function.

One can easily see that $E_{X}:\left(X, E_{X}\right) \rightarrow\left(X, E_{X}\right)$ is a fuzzy function and $E_{X}$ acts as identical morphism: if $R:\left(X, E_{X}\right) \rightarrow\left(Y, E_{Y}\right)$ and $S:\left(Y, E_{Y}\right) \rightarrow$ $\left(X, E_{X}\right)$ are fuzzy functions, then $R \circ E_{X}=R:\left(X, E_{X}\right) \rightarrow\left(Y, E_{Y}\right)$ and $E_{X} \circ S=S:\left(Y, E_{Y}\right) \rightarrow\left(X, E_{X}\right)$. Thus we obtain:

Proposition $4.3([20],[40])$. L-valued sets as objects and fuzzy functions as morphisms constitute a category denoted $\mathcal{F} \mathbf{S E T}(L)$.

In the sequel we will apply also the following properties of fuzzy functions:

Definition 4.4 ([40], [32]). Given a fuzzy function $R: X \times Y \rightarrow L$ we define the measure of its soundedness by

$$
\mu(R)=\bigwedge_{x} \bigvee_{y} R(x, y)
$$

A fuzzy function $R$ is called sound if $\mu(R)=1$.

Definition 4.5 (cf. [40], [32]). Given L-valued sets $\left(X, E_{X}\right)$ and $\left(Y, E_{Y}\right)$ and a fuzzy function $R: X \times Y \rightarrow L$, we define its degree of surjectivity by

$$
\sigma(R)=\bigwedge_{y} \bigvee_{x} R(x, y)
$$

A fuzzy function $R$ is called surjective if $\sigma(R)=1$.

The intuitive meaning of the value $\mu(R)$ is to what extent the set $X$ is the domain of the fuzzy function $R$. We can illustrate this by such example: Let $X, Y$ be sets, $X^{\prime} \subseteq X$ and $f: X^{\prime} \rightarrow Y$ be a function. Then interpreting $f$ as a fuzzy function $R_{f}:(X,=) \rightarrow(Y,=)$ where $L=\{0,1\}$, we have $\mu(f)=1$ iff $X^{\prime}=X$ and $\mu(f)=0$ otherwise.

The intuitive meaning of the value $\sigma(R)$ is the degree to which the set $Y$ is the range of the fuzzy function R. Interpreting a function $f: X \rightarrow Y$ as a fuzzy function $R_{f}:(X,=) \rightarrow(Y,=)$, we have $\sigma(f)=1$ if $f$ is surjective and $\sigma(f)=0$ otherwise.

It is proved in [40], see also [32] that if $R:\left(X, E_{X}\right) \rightarrow\left(Y, E_{Y}\right)$ and $S:\left(Y, E_{Y}\right) \rightarrow\left(Z, E_{Z}\right)$ are fuzzy functions, then $\mu(S \circ R) \geq \mu(R) * \mu(S)$ and 
$\sigma(S \circ R) \geq \sigma(R) * \sigma(S)$. Hence, in particular, composition of sound functions is sound and composition of surjective fuzzy functions is surjective.

Let $R: X \times Y \rightarrow L$ be a fuzzy function. Then referring to the generalization of the Zadeh's extension principle [48], we come to the following definition:

Definition 4.6 ([20], [40]). The forward power-set operator $R^{\rightarrow}: L^{X} \rightarrow$ $L^{Y}$ induced by the fuzzy function $R$ is defined by

$$
R \rightarrow(A)(y)=\bigvee_{x}(R(x, y) * A(x)) \forall A \in L^{X}, \forall y \in Y .
$$

Fuzzy set $R^{\rightarrow}(A) \in L^{Y}$ is called the image of the fuzzy set $A$ under the $L$ relation $R: X \times Y \rightarrow L$.

Definition 4.7 ([20], [40]). Let $R: X \times Y \rightarrow L$ be an L-relation. The backward power-set operator $R^{\leftarrow}: L^{Y} \rightarrow L^{X}$ induced by the fuzzy function $R$ is defined

$$
R^{\leftarrow}(B)(x)=\bigvee_{y} R(x, y) * B(y) \forall B \in L^{Y}, \forall x \in X
$$

Fuzzy set $R^{\leftarrow}(B) \in L^{X}$ is called the preimage of the fuzzy set $B \in L^{Y}$ under $L$-relation $R: X \times Y \rightarrow L$.

Proposition $4.8([40],[32])$. Let $\left(X, E_{X}\right),\left(Y, E_{Y}\right)$ be L-valued sets and $R:\left(X, E_{X}\right) \rightarrow\left(Y, E_{Y}\right)$ be a sound fuzzy function. Then:

(1) $R^{\rightarrow}\left(\bigvee_{i \in I}\left(A_{i}\right)\right)=\bigvee_{i \in I} R^{\rightarrow}\left(A_{i}\right) \quad \forall\left\{A_{i} \mid i \in I\right\} \subseteq L^{X}$;

(2) $R^{\rightarrow}\left(A_{1} \wedge A_{2}\right) \leq R^{\rightarrow}\left(A_{1}\right) \wedge R^{\rightarrow}\left(A_{2}\right) \forall A_{1}, A_{2} \in L^{X}$;

(3) $R^{\leftarrow}\left(\bigwedge_{i \in I} B_{i}\right)=\bigwedge_{i \in I}\left(R^{\leftarrow} B_{i}\right)$.

(4) $R^{\leftarrow}\left(\bigvee_{i \in I} B_{i}\right)=\bigvee_{i \in I}\left(R^{\leftarrow} B_{i}\right) \forall\left\{B_{i}: i \in I\right\} \subseteq L^{Y}$

(5) $A \leq R^{\leftarrow}\left(R^{\rightarrow}(A)\right) \forall A \in L^{X}$;

(6) $R^{\rightarrow}\left(R^{\leftarrow}(B)\right) \leq B$ in case $B$ is extensional.

(7) If $R$ is surjective and $B \in L^{Y}$ is extensional, then $\left(R^{\leftarrow}\right) \rightarrow(B)=B$;

(8) If $R$ is surjective, then $R^{\rightarrow}\left(\lambda_{X}\right)=\lambda_{Y}$ for every $\lambda \in L$.

REMARK 4.9. Note that if $f: X \rightarrow Y$ is an ordinary function, then the definitions of forward and backward powerset operators $R_{f}$ and $R_{f}^{\leftarrow}$ (see Remark 4.2) are actually the forward and backward powerset operators $f^{\rightarrow}$ : $L^{X} \rightarrow L^{Y}$ and $f^{\leftarrow}: L^{Y} \rightarrow L^{X}$ respectively, introduced and studied by S.E. Rodabaugh [34].

5. Category $\mathcal{F}$ BORN $(L, M)$ of $L M$-VAlued Bornological SPACES AND BOUNDED FUZZY FUNCTIONS

5.1. Bounded fuzzy functions of LM-valued bornological spaces.

Definition 5.1. Given two LM-valued bornological spaces $\left(X, E_{X}, \mathcal{B}_{X}\right)$ and $\left(Y, E_{Y}, \mathcal{B}_{Y}\right)$, we say that a fuzzy function $R:\left(X, E_{X}, \mathcal{B}_{X}\right) \rightarrow\left(Y, E_{Y}, \mathcal{B}_{Y}\right)$ is called bounded if $\mathcal{B}_{Y} \circ R \rightarrow \geq \mathcal{B}_{X}$. 
Explicitly the boundedness of a fuzzy function $R:\left(X, E_{X}, \mathcal{B}_{X}\right) \rightarrow$ $\left(Y, E_{Y}, \mathcal{B}_{Y}\right)$ means that $\mathcal{B}_{Y}\left(R^{\rightarrow}(A)\right) \geq \mathcal{B}_{X}(A)$ for every $A \in L^{X}$. One can easily prove the following

Proposition 5.2. Composition of two bounded fuzzy functions is bounded.

Since the identical fuzzy function $E_{X}:\left(X, E_{X}, \mathcal{B}_{X}\right) \rightarrow\left(X, E_{X}, \mathcal{B}_{X}\right)$ is bounded, we get

Proposition 5.3. LM-valued bornological spaces and bounded fuzzy functions form a category which will be denoted $\mathcal{F B O R N}(L, M)$.

Since composition of sound fuzzy functions is sound, we can distinguish a useful subcategory $\mathcal{F} \mathcal{S B O R N}$ of the category $\mathcal{F B O R N}$ whose objects are the same as in $\mathcal{F B O R N}$ and whose morphisms are sound fuzzy functions.

When studying properties of categories of $L M$-bornologies the construction of $L M$-bornologies from so called $L M$-valued bornology bases will be useful.

Definition 5.4. Let $\mathcal{L}$ be a subset of $L^{X}$ closed under finite unions and under taking extensional hulls. Then a mapping $\mathcal{C}: \mathcal{L} \rightarrow M$ is called an $L M$-valued bornology base if

(1LMBB) $\forall \alpha \in M^{\circ} \exists \mathcal{U} \subseteq \mathcal{L}$ such that $\bigvee \mathcal{U}=1_{L^{X}}$ and $\mathcal{C}(U) \geq \alpha \forall U \in \mathcal{U}$;

$(3 \mathrm{LMBB}) \mathcal{C}(A \vee B)=\mathcal{C}(A) \wedge \mathcal{C}(B) \forall A, B \in \mathcal{L}$;

(4LMBB) $\mathcal{C}(e(A))=\mathcal{C}(A) \forall A \in \mathcal{L}$ where $e(A)$ is the extensional hull of $A$.

Proposition 5.5. Let $\mathcal{C}: \mathcal{L} \rightarrow M$ be an LM-valued bornology base on $X$. By setting $\mathcal{B}_{\mathcal{C}}(A)=\bigvee\{\mathcal{C}(D): D \in \mathcal{L}, A \leq D\}$ we obtain an LM-valued bornology $\mathcal{B}_{\mathcal{C}}: L^{X} \rightarrow M$.

Proof. Axiom (1LMB) for $\mathcal{B}_{\mathcal{C}}$ follows directly from axiom (1LMBB) for $\mathcal{C}$. We obtain axiom (3LMB) for $\mathcal{B}_{\mathcal{C}}$ from the property (3LMBB) as follows:

$$
\begin{gathered}
\mathcal{B}_{\mathcal{C}}\left(A_{1} \vee A_{2}\right)=\bigvee\left\{\mathcal{C}(D): D \geq A_{1} \vee A_{2}\right\}=\bigvee\left\{\mathcal{C}\left(D_{1} \vee D_{2}\right): D_{1} \geq A_{1}, D_{2} \geq A_{2}\right\} \\
=\bigvee\left\{\mathcal{C}\left(D_{1}\right) \wedge \mathcal{C}\left(D_{2}\right): D_{1} \geq A_{1}, D_{2} \geq A_{2}\right\} \geq \\
\bigvee\left\{\mathcal{C}\left(D_{1}\right): D_{1} \geq A_{1}\right\} \wedge \bigvee\left\{\mathcal{C}\left(D_{2}\right): D_{2} \geq A_{2}\right\}=\mathcal{B}_{\mathcal{C}}\left(A_{1}\right) \wedge \mathcal{B}_{\mathcal{C}}\left(A_{2}\right)
\end{gathered}
$$

Axiom (2LMB) for $\mathcal{B}_{\mathcal{C}}$ follows from property (3LMBB) for $\mathcal{C}$ and already established (by (3LMB)) monotonicity of $\mathcal{B}_{\mathcal{C}}$.

Axiom (4LMB) for $\mathcal{B}_{\mathcal{C}}$ follows from property $(4 \mathrm{LMBB})$ for $\mathcal{C}$ and the assumptions on the $\mathcal{L}$.

From the definition of $\mathcal{B}_{\mathcal{C}}$, it is clear that $\mathcal{B}_{\mathcal{C}}: L^{X} \rightarrow M$ is the extension of the mapping $\mathcal{C}: \mathcal{L} \rightarrow M$ from $\mathcal{L}$ to $L^{X}$.

The proof of the following statement is straightforward: 
TheOREM 5.6. Let $\left(X, E_{X}, \mathcal{B}_{\mathcal{C}}\right)$ be an LM-valued bornological space, where $\mathcal{C}: \mathcal{L} \rightarrow M$ is a base for $L M$-valued bornology $\mathcal{B}_{\mathcal{C}}$. Then a fuzzy function $R:\left(X, E_{X}, \mathcal{B}_{\mathcal{C}}\right) \rightarrow\left(Y, E_{Y}, \mathcal{B}_{Y}\right)$ is bounded if and only if $\mathcal{B}_{Y}(R \rightarrow(A)) \geq \mathcal{B}_{X}(A)$ for every $A \in \mathcal{L}$.

\subsection{Preimages of LM-valued bornologies.}

THEOREM 5.7. Let $R:\left(X, E_{X}\right) \rightarrow\left(Y, E_{Y}, \mathcal{B}_{Y}\right)$ be a sound fuzzy function and let $\mathcal{L}:=\left\{A=R^{\leftarrow}(B) \mid B \in L^{Y}\right\}$. Then $\mathcal{C}_{X}=\mathcal{B}_{Y} \circ R^{\rightarrow}: \mathcal{L} \rightarrow M$ is a base for an $L M$-valued bornology $\mathcal{B}_{X}$ on $\left(X, E_{X}\right)$. Besides $\mathcal{B}_{X}$ is the coarsest LMvalued bornology for which the fuzzy function $R:\left(X, E_{X}, \mathcal{B}_{X}\right) \rightarrow\left(Y, E_{Y}, \mathcal{B}_{Y}\right)$. is bounded.

Proof. Note first that for every $B \in L^{Y}$ the preimage $R^{\leftarrow}(B)$ is extensional. Indeed $R^{\leftarrow}(B)(x) * E\left(x, x^{\prime}\right)=\bigvee_{y} R(x, y) * E\left(x, x^{\prime}\right) * B(y) \leq$ $\bigvee_{y} R\left(x^{\prime}, y\right) * B(y)=R^{\leftarrow} B\left(x^{\prime}\right)$.

In order to verify axiom (1LMBB) for the mapping $\mathcal{C}_{X}=\mathcal{B}_{Y} \circ R^{\rightarrow}$ : $\mathcal{L} \rightarrow M$, let $\alpha \in M^{\circ}$ and take a family $\mathcal{V}=\left\{V_{\xi} \mid \xi \in \Xi\right\} \subseteq L^{Y}$ such that $\bigvee_{\xi} V_{\xi}=1_{L}$ and $\mathcal{B}_{Y}\left(V_{\xi}\right) \geq \alpha$ for each $\xi \in \Xi$. Let

$$
\mathcal{U}=\left\{U_{\xi} \mid U_{\xi}=R^{\leftarrow}\left(V_{\xi}\right), V_{\xi} \in \mathcal{V}\right\},
$$

then $\mathcal{C}_{X}\left(U_{\xi}\right) \geq \alpha$ for all $\xi \in \Xi$. Besides the family $\mathcal{U}$ is an (extensional) cover of the set $(X, E)$. Indeed, given a point $x \in X$ we have:

$$
\begin{gathered}
\bigvee_{\xi \in \Xi} U_{\xi}(x)=\bigvee_{\xi \in \Xi} R^{\leftarrow}\left(V_{\xi}\right)(x)=\bigvee_{\xi \in \Xi}\left(\bigvee_{y \in Y} R(x, y) * V_{\xi}(y)\right) \\
=\bigvee_{y \in Y}\left(R(x, y) * \bigvee_{\xi \in \Xi} V_{\xi}(y)\right)=\bigvee_{y \in Y}\left(R(x, y) * 1_{L}\right)=\bigvee_{y \in Y} R(x, y)=1_{L} ;
\end{gathered}
$$

the last equality is ensured by soundedness of the fuzzy function $R$.

To prove axiom (3LMBB) let $A_{1}=R^{\leftarrow}\left(B_{1}\right), A_{2}=R^{\leftarrow}\left(B_{2}\right)$ Then from the definition of $\mathcal{C}_{X}$ and applying Proposition 4.8 we have

$$
\begin{gathered}
\mathcal{C}_{X}\left(A_{1}\right) \wedge \mathcal{C}_{X}\left(A_{2}\right)=\mathcal{B}_{Y}\left(B_{1}\right) \wedge \mathcal{B}_{Y}\left(B_{2}\right)=\mathcal{B}_{Y}\left(B_{1} \vee B_{2}\right) \\
=\mathcal{C}_{X}\left(R^{\leftarrow}\left(B_{1} \vee B_{2}\right)\right)=\mathcal{C}_{X}\left(R^{\leftarrow}\left(B_{1}\right) \vee R^{\leftarrow}\left(B_{2}\right)\right)=\mathcal{C}_{X}\left(A_{1} \vee A_{2}\right)
\end{gathered}
$$

To show (4MLBB) recall that if $A \in \mathcal{L}$, then $A=e(A)$ (as the preimage of fuzzy set under a fuzzy function) and hence $\mathcal{C}_{X}(A)=\mathcal{C}_{X}(e(A))$.

To conclude the proof note that from the construction it is clear that $\mathcal{B}_{X}$ is the coarsest $L M$-valued bornology on $\left(X, E_{X}\right)$, for which $R:\left(X, E_{X}, \mathcal{B}_{X}\right) \rightarrow$ $\left(Y, E_{Y}, \mathcal{B}_{Y}\right)$ is bounded.

We call the $L M$-valued bornology $\mathcal{B}_{X}$ constructed above the preimage of the $L M$-valued bornology $\mathcal{B}_{Y}$ under fuzzy function $R:\left(X, E_{X}\right) \rightarrow\left(Y, E_{Y}, \mathcal{B}_{Y}\right)$ and denote it by $R^{\leftarrow}\left(\mathcal{B}_{Y}\right)$. 
5.3. Initial LM-valued bornologies induced by families of sound fuzzy functions.

TheOrem 5.8. For every family $R_{i}:\left(X, E_{X}\right) \rightarrow\left(Y_{i}, E_{Y_{i}}, \mathcal{B}_{Y_{i}}\right)$ of sound fuzzy functions, there exists the coarsest $L M$-valued bornology $\mathcal{B}_{X}$ for which all fuzzy functions $R_{i}:\left(X, E_{X}, \mathcal{B}_{X}\right) \rightarrow\left(Y_{i}, E_{Y_{i}}, \mathcal{B}_{Y_{i}}\right)$ are bounded.

Proof. Given a family of sound fuzzy functions

$$
R_{i}:\left(X, E_{X}\right) \rightarrow\left(Y_{i}, E_{Y_{i}} \mathcal{B}_{Y_{i}}\right), i \in I,
$$

we define the $L M$-valued bornology on the set $X$ by setting $\mathcal{B}_{X}=\curlywedge_{i \in I} \mathcal{B}_{i}^{X}$, where $\mathcal{B}_{i}^{X}=R_{i}^{\leftarrow} \mathcal{B}_{Y_{i}}$ is the preimage of the $L M$-valued bornology $\mathcal{B}_{Y_{i}}$ by the fuzzy function $R_{i}$ and $\curlywedge$ is the infimum in the lattice ( $\left.\mathfrak{B}(X, E, L, M), \preceq, \curlywedge, \curlyvee\right)$, Referring to Section 3.2 we conclude that $\mathcal{B}_{X}$ is an $L M$-valued bornology on the $L$-valued set $\left(X, E_{X}\right)$; besides it is the coarsest one in the lattice $(\mathfrak{B}(X, E, L, M), \preceq, \curlywedge, \curlyvee)$ of all $L M$-valued bornologies $\mathcal{B}_{X}^{\prime}$ on $\left(X, E_{X}\right)$ for which all fuzzy functions $R_{i}:\left(X, E_{X}, \mathcal{B}_{X}^{\prime}\right) \rightarrow\left(Y, E_{Y_{i}}, \mathcal{B}_{Y_{i}}\right)$ are bounded.

Applying terminology from the category theory we easily get the following statement from the previous theorem:

TheOrem 5.9. Every source $R_{i}:\left(X, E_{X}\right) \rightarrow\left(Y_{i}, E_{Y_{i}}, B_{Y_{i}}\right), i \in I$ has a unique initial lift $R_{i}:\left(X, E_{X}, \mathcal{B}_{X}\right) \rightarrow\left(Y_{i}, E_{Y_{i}}, B_{Y_{i}}\right), i \in I$ in the category

$\mathcal{F S B O R N}(L, M))$ of sound LM-valued bornological spaces.

The existence of initial $L M$-valued bornologies implies also the following

Corollary 5.10. Products exist in the category $\mathcal{F S B O R N}(L, M))$.

\subsection{Images of LM-bornologies.}

TheOREM 5.11. Let $\left(X, E_{X}, \mathcal{B}_{X}\right)$ be an LM-valued bornological space, $\left(Y, E_{Y}\right)$ be an $L$-valued set and let $R:\left(X, E_{X}, \mathcal{B}_{X}\right) \rightarrow\left(Y, E_{Y}\right)$ be a surjective sound fuzzy function. By setting $\mathcal{B}_{Y}=\mathcal{B}_{X} \circ R^{\leftarrow}: L^{Y} \rightarrow M$ an LM-valued bornology on $\left(Y, E_{Y}\right)$ is defined. Besides, it is the finest LM-valued bornology on $\left(Y, E_{Y}\right)$ for which fuzzy function $R:\left(X, E_{X}, \mathcal{B}_{X}\right) \rightarrow\left(Y, E_{Y}, \mathcal{B}_{Y}\right)$ is bounded. We call $\mathcal{B}_{Y}:=R^{\rightarrow}\left(\mathcal{B}_{X}\right)$ the image of the LM-valued bornology $\mathcal{B}_{X}$ under the surjective fuzzy function $R:\left(X, E_{X}, \mathcal{B}_{X}\right) \rightarrow\left(Y, E_{Y}\right)$.

Proof. Let $R:\left(X, E_{X}, \mathcal{B}_{X}\right) \rightarrow\left(Y, E_{Y}\right)$ be a surjective fuzzy function from an $L M$-bornological space $\left(X, E_{X}, \mathcal{B}_{X}\right)$ to an $L$-valued set $\left(Y, E_{Y}\right)$ and let a mapping $\mathcal{B}_{Y}: L^{Y} \rightarrow M$ be defined by $\mathcal{B}_{Y}=\mathcal{B}_{X} \circ R^{\leftarrow}: L^{Y} \rightarrow M$. We claim that $\mathcal{B}_{Y}$ is an $L M$-valued bornology on $\left(Y, E_{Y}\right)$, and besides it is the finest $L M$-valued bornology $\mathcal{B}_{Y}$ on the $L$-valued set $\left(Y, E_{Y}\right)$ for which the fuzzy function $R:\left(X, E_{X}, \mathcal{B}_{X}\right) \rightarrow\left(Y, E_{Y}, \mathcal{B}_{Y}\right)$ is bounded.

To verify axiom (1LMB) for $\mathcal{B}_{Y}$ we fix $\alpha \in M^{\circ}$. Referring to axiom (1LMB) in case of the $L M$-valued bornology $\mathcal{B}_{X}$ find a family of $L$-fuzzy sets $\mathcal{U} \subseteq L^{X}$ such that $\mathcal{B}_{X}(U) \geq \alpha$ for every $U \in \mathcal{U}$ and $\bigvee\{\mathcal{U} \mid U \in \mathcal{U}\}=1_{L^{X}}$. 
For every $U \in \mathcal{U}$ let $V_{U}=R^{\rightarrow}(U)$ and let $\mathcal{V}=\left\{V_{U} \mid U \in \mathcal{U}\right\}$. Then, taking into account that $R:\left(X, E_{X}, \mathcal{B}_{X}\right) \rightarrow\left(Y, E_{Y}\right)$ is surjective and sound and applying Proposition 4.8, we have

$$
\begin{gathered}
\bigvee\left\{V_{U} \mid U \in \mathcal{U}\right\}=\bigvee\left\{R^{\rightarrow}(U) \mid U \in \mathcal{U}\right\}=R^{\rightarrow}(\bigvee\{U \mid U \in \mathcal{U}\}) \\
=R^{\rightarrow}\left(1_{L^{X}}\right)=1_{L^{Y}} .
\end{gathered}
$$

Since, by definition of $\mathcal{B}_{Y}$, for every $V_{U} \in \mathcal{V}$ it holds $\mathcal{B}_{Y}\left(V_{U}\right)=\mathcal{B}_{X}(U) \geq \alpha$, the validity of axiom (1LMB) for $\mathcal{B}_{Y}$ is established.

The validity of the property $(2 \mathrm{LMB})$ for $\mathcal{B}_{Y}$ follows directly since $\mathcal{B}_{X}$ has this property. To establish property (3LMB), we refer to Proposition 4.8 and are reasoning as follows:

$$
\begin{gathered}
\mathcal{B}_{Y}\left(B_{1} \vee B_{2}\right)=\mathcal{B}_{X}\left(R^{\leftarrow}\left(B_{1} \vee B_{2}\right)\right) \\
\geq \mathcal{B}_{X}\left(R^{\leftarrow}\left(B_{1}\right)\right) \wedge \mathcal{B}_{X}\left(R^{\leftarrow}\left(B_{2}\right)\right)=\mathcal{B}_{Y}\left(B_{1}\right) \wedge \mathcal{B}_{Y}\left(B_{2}\right)
\end{gathered}
$$

The validity of the property (4LMB) for $\mathcal{B}_{Y}$ follows straightforward from property (4LMB) for $\mathcal{B}_{X}$ and the general statement established in Proposition 5.12 below.

Besides, from the construction it is easy to notice that $\mathcal{B}_{Y}$ is the finest $L M$-valued bornology for which $R$ is bounded, that is the final $L M$-valued bornology for the fuzzy function $R:\left(X, \mathcal{B}_{X}, E_{X}\right) \rightarrow\left(Y, E_{Y}\right)$.

Proposition 5.12. Let $R:\left(X, E_{X}\right) \rightarrow\left(Y, E_{Y}\right)$ be a fuzzy function. Then the image $R^{\rightarrow}(A)$ is extensional for every $A \in L^{X}$. In particular, $e\left(R^{\rightarrow}(A)\right)=$ $R \rightarrow(e(A))$.

Proof. The proof follows from the next sequence of equalities:

$$
\begin{gathered}
R^{\rightarrow}(A)(y) * E_{Y}\left(y, y^{\prime}\right)=\bigvee_{x \in X} R(x, y) * A(x) * E\left(y, y^{\prime}\right) \\
\leq \bigvee_{x \in X} R\left(x, y^{\prime}\right) * A(x)=R^{\rightarrow}(A)\left(y^{\prime}\right)
\end{gathered}
$$

Let now $R:\left(X, \mathcal{B}_{X}, E_{X}\right) \rightarrow\left(Y, E_{Y}\right)$ be an arbitrary sound fuzzy function, and let the image $R \rightarrow\left(\mathcal{B}_{X}\right)$ of the $L M$-valued bornology $\mathcal{B}_{X}$ for it be defined in the same way as in Theorem 5.11. Analyzing the proof of this theorem we can notice that the surjectiveness of the fuzzy function $R:\left(X, \mathcal{B}_{X}, E_{X}\right) \rightarrow\left(Y, E_{Y}\right)$ was used only in the proof of the property (1LMB); the proofs of all other properties of $R \rightarrow\left(\mathcal{B}_{X}\right)$ were obtained either for any fuzzy function $R:\left(X, \mathcal{B}_{X}, E_{X}\right) \rightarrow\left(Y, E_{Y}\right)$ or assuming only its soundedness. So, to get the initial $L M$-valued fuzzy bornology induced by a fuzzy function $R:\left(X, \mathcal{B}_{X}, E_{X}\right) \rightarrow\left(Y, E_{Y}\right)$ we have to "strengthen" in the "optimal" way $R \rightarrow\left(\mathcal{B}_{X}\right)$ in order to achieve property (1LMB) and not spoiling other properties of $R \rightarrow\left(\mathcal{B}_{X}\right)$. One can easily notice that this can be obtained 
by means of the finest $L M$-valued bornology $\mathcal{B}_{Y}^{\top}$ on $\left(Y, E_{Y}\right)$ and in the result get the following

TheOREM 5.13. Let $R:\left(X, E_{X}, \mathcal{B}_{X}\right) \rightarrow\left(Y, E_{Y}\right)$ be a sound fuzzy function. Then by setting $\mathcal{B}_{Y}=R^{\rightarrow}\left(\mathcal{B}_{X}\right) \curlywedge \mathcal{B}_{Y}^{\top}$ we get the final LM-valued bornology $\mathcal{B}_{Y}$ on $\left(Y, E_{Y}\right)$, that is the finest bornology on $\left(Y, E_{Y}\right)$ for which the sound fuzzy function $R:\left(X, E_{X}, \mathcal{B}_{X}\right) \rightarrow\left(Y, E_{Y}, \mathcal{B}_{Y}\right)$ is bounded.

5.5. Final LM-valued bornologies induced by families of sound fuzzy functions.

Let now $\left\{R_{i}:\left(X_{i}, E_{X_{i}}, \mathcal{B}_{X_{i}}\right) \rightarrow\left(Y, E_{Y}\right) \mid i \in I\right\}$ be a family of sound fuzzy functions. For every $i \in I$ let $\mathcal{B}_{Y}^{i}: L^{Y} \rightarrow M$ be the final $L M$-valued bornology induced by the fuzzy function $R_{i}:\left(X_{i}, E_{X_{i}}, \mathcal{B}_{X_{i}}\right) \rightarrow\left(Y, E_{Y}\right)$. Further, let $\mathcal{B}_{Y}:=\curlywedge_{i \in I} \mathcal{B}_{Y}^{i}: L^{Y} \rightarrow M$ where $\curlywedge$ is the infimum in the lattice of all $L M$ valued bornologies on the $L$-valued set $\left(Y, E_{Y}\right)$. Analyzing the construction one can easily make confident in the following Theorem:

TheOREM 5.14. By setting $\mathcal{B}_{Y}:=\curlywedge_{i \in I} \mathcal{B}_{Y}^{i}: L^{Y} \rightarrow M$ we define the finest $L M$-valued bornology on the $L$-valued set $\left(Y, E_{Y}\right)$ for which all sound fuzzy functions $R_{i}$ are bounded. Or, say it in a different way, $\mathcal{B}_{Y}:=$ $\curlywedge_{i \in I} \mathcal{B}_{Y}^{i}: L^{Y} \rightarrow M$ is the final LM-valued bornology induced by the family $\left\{R_{i}:\left(X_{i}, E_{X_{i}}, \mathcal{B}_{X_{i}}\right) \rightarrow\left(Y, E_{Y}\right) \mid i \in I\right\}$ of sound fuzzy functions. ing

Applying the terminology of the category theory we easily get the follow-

TheOREM 5.15. Every sink $R_{i}:\left(X_{i}, E_{X_{i}}, \mathcal{B}_{X_{i}}\right) \rightarrow\left(Y, E_{Y}\right), i \in I$ in the category $\mathcal{F} \mathcal{S B O R N}(L, M)$ has a unique final lift $R_{i}:\left(X_{i}, E_{X_{i}}, \mathcal{B}_{X_{i}}\right) \rightarrow$ $\left(Y, E_{Y}, \mathcal{B}_{Y}\right), i \in I$.

COROllary 5.16. Co-products and quotients exist in the category $\mathcal{F S B O R N}(L, M)$.

\section{Subcategories of the Category $\mathcal{F} \mathcal{S B O R N}(L, M)$}

6.1. Category $\mathcal{F} \mathcal{S B O R N}(L, 2)$.

Let $M$ be the two-element lattice $2=\{0,1\}$ and let $E: X \times X \rightarrow L$ be an $L$-valued equality on $X$. Then the $L 2$-valued bornology on the $L$-valued set $X$ is just a family $\mathcal{B} \subseteq L^{X}$ such that

(1L2B) $\bigvee\{A \mid A \in \mathcal{B}\}=1_{L}$

(2L2B) $B \leq A, A \in \mathcal{B} \Rightarrow B \in \mathcal{B}$;

(3L2B) $A_{1}, A_{2} \in \mathcal{B} \Rightarrow A_{1} \vee A_{2} \in \mathcal{B}$;

(4L2B) $A \in \mathcal{B} \Longrightarrow e(A) \in \mathcal{B}$.

We refer to objects of $\mathcal{F S B O R N}(L, 2)$ as $L$-fuzzy bornological spaces. In case axiom $(1 \mathrm{~L} 2)$ is replaced by a stronger axiom

$\left(1^{\prime}\right.$ L2) $\{x\} \in \mathcal{B}$ for every $x \in X$ 
we get the category $\mathcal{F S}^{s} \mathbf{B O R N}(L, 2)$ of strong $L$-fuzzy bornological spaces Now, let $\mathbf{B O R N}(L, 2)$ and $\mathbf{B O R N}^{s}(L, 2)$ be the subcategories of the category $\mathcal{F S B O R N}(L, 2)$ and $\mathcal{F} \mathcal{S}^{s} \mathbf{B O R N}(L, 2)$ respectively, whose objects are $L 2$-bornological spaces on $(X,=)$, that is $X$ is an ordinary set, and whose morphisms are fuzzy functions $R_{f}:\left(X,=_{X}, \mathcal{B}_{X}\right) \rightarrow\left(Y,=_{Y}, \mathcal{B}_{Y}\right)$ determined by ordinary functions $f: X \rightarrow Y$ (see Remark 4.2) and such that $\mathcal{B}_{Y}(f(A)) \geq \mathcal{B}_{X}(A)$. The categories $\operatorname{BORN}(L, 2)$ and $\operatorname{BORN}^{s}(L, 2)$ thus obtained are actually the categories of $L$-bornological spaces and strong $L$ bornological spaces, see Subsection 1.2.

6.2. Category $\mathcal{F} \mathcal{S B O R N}(2, M)$.

Let $L=2$ be the two-element lattice. Then $2^{X}$ is just the family of all subsets of a set $X$. Further, let $X$ be considered with a crisp equality $=$. Then the $2 M$-valued bornology on $X$ is a mapping $\mathcal{B}: 2^{X} \rightarrow M$ such that

(1 2MB) $\mathcal{B}(\{x\})=1_{M}$ for every $x \in X$;

(2 2MB) $A \subseteq B \Rightarrow \mathcal{B}(B) \leq \mathcal{B}(A) \forall A, B \in \mathbb{B}$;

(3 2MB) $\mathcal{B}(A \cap B) \geq \mathcal{B}(A) \wedge \mathcal{B}(B) \forall A, B \in \mathbb{B}$

Now, let $\operatorname{BORN}(2, M)$ be the subcategory of $\mathcal{F} \mathcal{S B O R N}(2, M)$ whose objects are $2 M$-valued bornological spaces and whose morphisms are fuzzy functions $R_{f}:\left(X,=_{X}, \mathcal{B}_{X}\right) \rightarrow\left(Y,=_{Y}, \mathcal{B}_{Y}\right)$ determined by ordinary functions $f: X \rightarrow Y$ and such that $\mathcal{B}_{Y}(f(A)) \geq \mathcal{B}_{X}(A)$. The category $\operatorname{BORN}(2, M)$ thus obtained is actually the category of $M$-valued bornological spaces see Subsection 1.3.

\section{7. $L M$-VALUED MODIFICATION OF AN $L$-BORNOLOGY ON A METRIC SPACE}

In this section, we develop a construction illustrating how new $L M$-valued bornologies can be constructed. Namely, we present a construction of an $L M$ valued bornology on a set $X$ from an arbitrary $L$-bornology on a metric space $(X, \rho)$. Among other, the proposed construction allows to get a deeper insight into a bornology using the tools of its fuzzification.

7.1. A family of L-valued equalities generated by a metric.

Let $L=M=[0,1]$ be the unit interval viewed as a quantale $([0,1], \leq, \wedge, \vee, *)$ where $*: L \times L \rightarrow L$ is a continuous $t$-norm (see e.g. [24]). Further, let $X$ be a set and $\rho: X \times X \rightarrow[0,1]$ be a metric. For every $\alpha \in[0,1]$ we define a mapping $E_{\rho}^{\alpha}: X \times X \rightarrow[0,1]$ by setting

$$
E_{\rho}^{\alpha}(x, y)=\left\{\begin{aligned}
\frac{1-\alpha}{1-\alpha+\alpha \rho(x, y)} & \text { if } \alpha \neq 1 \text { or } \rho(x, y) \neq 0 \\
1 & \text { if } \alpha=1 \text { and } \rho(x, y)=0 .
\end{aligned}\right.
$$

We compare the obtained mappings $E_{\rho}^{\alpha}: X \times X \rightarrow L=[0,1]$ with the properties of the $L$-valued relations, see subsection 2.3: 
Proposition 7.1. For every metric $\rho: X \times X \rightarrow[0,1]$ and every $\alpha \in M=[0,1]$ the mapping $E_{\rho}^{\alpha}: X \times X \rightarrow[0,1]$ satisfies conditions (1E) (reflexivity) and (2E) (symmetricity). In cases of the product t-norm $*=\cdot$ (and hence for every weaker $t$-norm) $E_{\rho}^{\alpha}$ satisfies condition (3E) that is transitive. If $\rho$ is an ultra-metric, then mapping $E_{\rho}^{\alpha}: X \times X \rightarrow[0,1]$ is transitive in case of the minimum $t$-norm $*=\wedge$, and hence in case of any continuous $t$-norm.

The validity of conditions (1E) and (2E) follows directly from the definition of the mapping $E_{\rho}: X \times X \times[0,1] \rightarrow[0,1]$.

To prove (3E) consider separately the cases of the two t-norms:

$*=\wedge$ Since in this case $\rho$ is assumed to be an ultra pseudo-metric, we have $\rho(x, y) \leq \max \{\rho(x, z), \rho(z, y)\}$ for all $x, y, z$. It is straightforward to conclude from here that

$$
\frac{1-\alpha}{1-\alpha+\alpha \rho(x, y)} \geq \frac{1-\alpha}{1-\alpha+\alpha \rho(x, z)} \bigwedge \frac{1-\alpha}{1-\alpha+\alpha \rho(z, y)} .
$$

$*=$. The inequality

$$
\frac{1-\alpha}{1-\alpha+\alpha \rho(x, y)} \geq \frac{1-\alpha}{1-\alpha+\alpha \rho(x, z)} \cdot \frac{1-\alpha}{1-\alpha+\alpha \rho(z, y)}
$$

can be easily established taking into account the triangular property $\rho(x, y) \leq \rho(x, z)+\rho(z, y)$ of the metric $\rho$.

Having fixed $x, y \in X$ and changing $\alpha \in M$ we may consider a function $E(x, y): M \rightarrow M$ by setting $E(x, y)(\alpha)=E^{\alpha}(x, y)$. The proof of the following proposition is clear from the definition of the family $\left\{E^{\alpha}: \alpha \in M\right\}$ :

Proposition 7.2. For every $(x, y) \in X \times X$ the mapping $E(x, y): M \rightarrow$ $M$ is continuous.

Concerning the interrelation of the constructed $L$-valued equalities $E^{\alpha}$ for different $\alpha \in M$ we have the following

Proposition 7.3.

$(4 E) \alpha \leq \beta \Longrightarrow E^{\alpha}(x, y) \geq E^{\beta}(x, y)$ for all $x, y \in X, \alpha, \beta \in M$;

(5E) $E_{\rho} \bar{\bigvee}_{n \in \mathbb{N}}^{\alpha_{n}}(x, y)=\bigwedge_{n \in \mathbb{N}} E_{\rho}^{\alpha_{n}}(x, y)$ for all $x, y \in X,\left\{\alpha_{n} \mid n \in \mathbb{N}\right\} \subseteq M$;

(6E) $E_{\rho}^{\bigwedge_{n \in \mathbb{N}}^{\alpha_{n}}}(x, y)=\bigvee_{n \in \mathbb{N}} E_{\alpha_{n}}(x, y)$ for all $x, y \in X,\left\{\alpha_{n} \mid n \in \mathbb{N}\right\} \subseteq M$;

$(7 E) E_{\rho}^{0_{M}}(x, y)=1_{L}$ for all $x, y \in X$;

$$
E_{\rho}^{1_{M}}(x, y)= \begin{cases}1_{L} & \text { if } x=y \\ 0_{L} & \text { otherwise. }\end{cases}
$$

Proof. Property ( $4 E$ ) follows directly from the definition of $E_{\rho}^{\alpha}$.

To prove Property $(5 E)$ let $\alpha=\bigvee_{n \in \mathbb{N}} \alpha_{n}$. Without loss of generality we may assume that $n \leq n+1 \Rightarrow \alpha_{n} \leq \alpha_{n+1}$ for every $n \in \mathbb{N}$. Then, referring to 
Proposition 7.2 and (4E), we have

$$
E_{\rho}^{\alpha}(x, y)=E_{\rho}^{\bigvee_{n} \alpha_{n}}(x, y)=\lim _{n \rightarrow \infty} E_{\rho}^{\alpha_{n}}(x, y,)=\bigwedge_{n \in \mathbb{N}} E_{\rho}^{\alpha_{n}}(x, y)
$$

To prove Property $(6 E)$ let $\alpha=\bigwedge_{n \in \mathbb{N}} \alpha_{n}$. Without loss of generality we may assume that $n \leq n+1 \Rightarrow \alpha_{n} \geq \alpha_{n+1}$ for every $n \in \mathbb{N}$. Then, referring to Proposition 7.2 and property (4E) we have

$$
E_{\rho}^{\alpha}(x, y)=E_{\rho}^{\bigwedge_{n}^{\alpha_{n}}}(x, y)=\lim _{n \rightarrow \infty} E_{\rho}^{\alpha_{n}}(x, y)=\bigvee_{n \in \mathbb{N}} E_{\rho}^{\alpha_{n}}(x, y) .
$$

From the definition of $E_{\rho}$ it is clear that $E_{\rho}^{0_{M}}(x, y)=1$ for every $x, y \in X$ and

$$
E_{\rho}^{1_{M}}(x, y)= \begin{cases}1_{L} & \text { if } x=y \\ 0_{L} & \text { otherwise }\end{cases}
$$

and hence the properties $(7 E)$ and $(8 E)$ hold.

Let an $L$-fuzzy set $A \in L^{X}$ and $\alpha \in M$ be given. Then by $e^{\alpha}(A)$ we denote the extensional hull of $A$ in the $L$-valued set $\left(X, E_{\alpha}\right)$, that is $e^{\alpha}(A)(x)=$ $\bigvee_{x^{\prime} \in X}\left(E_{\alpha}\left(x, x^{\prime}\right) * A\left(x^{\prime}\right)\right)$. In the result for each $\alpha \in M$ we obtain an operator $e^{\alpha}: L^{X} \rightarrow L^{X}$. Concerning the properties of the family $e^{\alpha}: L^{X} \rightarrow L^{\prime} \alpha \in M$ we have the following proposition:

Proposition 7.4. Operators $e^{\alpha}: L^{X} \rightarrow L^{X}$ have following properties:

(1e) $e^{\alpha}(A) \geq A$ for every $A \in L^{X}$, for every $\alpha \in M$;

(2e) $e^{\alpha}\left(\bigvee_{\lambda \in \Lambda} A_{\lambda}\right)=\bigvee_{\lambda \in \Lambda} e^{\alpha}\left(A_{\lambda}\right)$ for any family $\left\{A_{\lambda} \mid \lambda \in \Lambda\right\} \subseteq L^{X}$.

In particular $e^{\alpha}(A \vee B)(x)=e^{\alpha}(A)(x) \vee e^{\alpha}(B)(x) \forall x \in X, \alpha \in M$.

(3e) $\alpha \leq \beta \Rightarrow e^{\alpha}(A)(x) \geq e^{\beta}(A)(x) \forall x \in X$;

(4e) $e^{\bigvee_{n}^{\alpha_{n}}}(A)(x)=\bigwedge_{n} e^{\alpha_{n}}(A)(x)$ for every sequence $\left\{\alpha_{n} \mid n \in \mathbb{N}\right\} \subseteq M$;

(5e) $e^{\bigwedge_{n}{ }^{\alpha_{n}}}(A)(x)=\bigvee_{n} e^{\alpha_{n}}(A)(x)$ for every sequence $\left\{\alpha_{n} \mid n \in \mathbb{N}\right\} \subseteq M$;

(6e) $e^{\alpha}\left(e^{\alpha}(A)\right)=e^{\alpha}(A)$;

(7e) $e^{0_{M}}(A)(x)=\bigvee_{x^{\prime} \in X} A\left(x^{\prime}\right)$;

(8e) $e^{1_{M}}(A)(x)=A(x)$.

Proof. The first property is ensured by the reflexivity of the relation $E^{\alpha}: X \times X \rightarrow L$. Property (2e) is clear from the definition of $e^{\alpha}$. Property (3e) follows from the established implication $\alpha \leq \beta \Rightarrow E^{\alpha}(x, y) \geq E^{\beta}(x, y)$.

We establish property $(4 \mathrm{e})$ as follows:

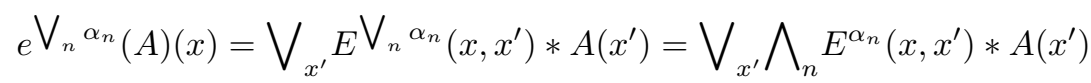

$$
\begin{aligned}
& \leq \bigwedge_{n} \bigvee_{x^{\prime}} E^{\alpha_{n}}\left(x, x^{\prime}\right) * A\left(x^{\prime}\right)=\bigwedge_{n} e^{\alpha_{n}}(A)(x)
\end{aligned}
$$

To show the converse inequality, assume that there exists $\beta$ such that

$$
\bigvee_{x^{\prime}} \bigwedge_{n} E^{\alpha_{n}}\left(x, x^{\prime}\right) * A\left(x^{\prime}\right)<\beta<\bigwedge_{n} \bigvee_{x^{\prime}} E^{\alpha_{n}}\left(x, x^{\prime}\right) * A\left(x^{\prime}\right)
$$


Then for each $x^{\prime}$ there exists $n_{0}$ such that $E^{\alpha_{n_{0}}}\left(x, x^{\prime}\right) * A\left(x^{\prime}\right)<\beta$. However knowing that the family of $L$-valued equalities is non-increasing and from the definition of the sequence $\alpha_{n}$ we may assume that $E^{\alpha_{n}}\left(x, x^{\prime}\right) * A\left(x^{\prime}\right)<\beta$ for all $n \in \mathbb{N}$. Hence also $\bigwedge_{n} \bigvee_{x^{\prime}} E^{\alpha_{n}}\left(x, x^{\prime}\right) * A\left(x^{\prime}\right)<\beta$. The obtained contradiction completes the proof of the property $(4 \mathrm{e})$

We establish property (5e) as follows:

$$
\begin{aligned}
e^{\bigwedge_{n}{ }^{\alpha_{n}}(A)(x)} & =\bigvee_{x^{\prime}}{ }^{\Lambda_{n}^{\alpha_{n}}}\left(x, x^{\prime}\right) * A\left(x^{\prime}\right)=\bigvee_{x^{\prime}} \bigvee_{n} E^{\alpha_{n}}\left(x, x^{\prime}\right) * A\left(x^{\prime}\right) \\
= & \bigvee_{n} \bigvee_{x^{\prime}} E^{\alpha_{n}}\left(x, x^{\prime}\right) * A\left(x^{\prime}\right)=\bigvee_{n} e^{\alpha_{n}}(A)(x)
\end{aligned}
$$

Property (6e) follows from the fact that $e^{\alpha}(A)$ is the extensional hull of $A$ in the $L$-valued set $(X, E)$.

Properties (7e) and (8e) follow from properties (7E) and (8E) of Proposition 7.2 .

REMARK 7.5. The construction presented in this subsection was done in case of a metric $\rho: X \times X \rightarrow[0,1]$, that is in case when the metric that takes its values in the unit interval $[0,1]$. We can modify the construction in order to apply it for any metric $d: X \times X \rightarrow \mathbb{R}^{+}=[0, \infty)$ by replacing the original metric $d$ with the metric $\rho: X \times X \rightarrow[0,1)$ defined by $\rho(x, y)=\frac{d(x, y)}{1+d(x, y)}$. It is known that metrics $d$ and $\rho$ induce the same topology on the set $X$, see, e.g. [10]. Note also that a set $A$ is bounded in the original metric $d$ if and only if $\operatorname{diam}_{\rho}(A)<1$ in metric $\rho$.

7.2. LM-valued modification of an L-bornology on a set $X$ by means of a metric $\rho: X \times X \rightarrow \mathbb{R}^{+}$.

Let, as before $L=M=[0,1], X$ be a set and $\rho: X \times X \rightarrow \mathbb{R}^{+}$be a metric. Referring to Remark 7.5, without loss of generality we may assume that $\rho$ takes its values in the interval $[0,1]$. Further, let $E^{\alpha}: X \times X \rightarrow L$ and $e^{\alpha}: L^{X} \rightarrow L^{X}$ be the $L$-valued equality and the operator of extensional hull defined as above. Given an $L$-bornology ${ }^{3} \mathbb{B} \subseteq L^{X}$ on a set $X$ and applying notations introduced above we define two families of $L$-fuzzy subsets of $X$ by setting $\mathbb{B}_{\alpha}=\left\{e^{\alpha}(A): A \in \mathbb{B}\right\}$ and $\overline{\mathbb{B}}_{\alpha}=\left\{P \in L^{X}: \exists A \in \mathbb{B}\right.$ such that $P \leq$ $\left.e^{\alpha}(A)\right\}$.

Proposition 7.6. For each $\alpha$ the family $\mathbb{B}_{\alpha}$ is an L-fuzzy bornology base on the L-valued set $\left(X, E_{\alpha}\right)$ and the family $\overline{\mathbb{B}}_{\alpha}$ is the corresponding L-fuzzy bornology, see Subsection 6.1.

Proof. Indeed, the validity of axiom (1L2B) for $\mathbb{B}^{\alpha}$ is obvious from the properties $(1 \mathrm{LB})$ of the original $L$-bornology $\mathbb{B}$. To show axiom $(3 \mathrm{~L} 2 \mathrm{~B})$

\footnotetext{
${ }^{3}$ specifically it can be the bornology induced by the metric $\rho$
} 
for $\mathbb{B}^{\alpha}$ it is sufficient to notice that from the properties of the hull operator $e^{\alpha}: L^{X} \rightarrow L^{X}$ we have

$$
e^{\alpha}(A) \wedge e^{\alpha}(B)=e^{\alpha}(A \wedge B) \text { for all } A, B \in L^{X} .
$$

Finally, recalling the property $(6 \mathrm{e})$ of the operator $e^{\alpha}$ it holds $e^{\alpha}(A)=A$ for all $A \in \mathbb{B}^{\alpha}$ and hence (4L2B) holds for $\mathbb{B}^{\alpha}$. Thus $\mathbb{B}^{\alpha}$ is an $L$-fuzzy bornology base. From here and taking into account that the validity of the axiom (2L2B) for $\overline{\mathbb{B}}_{\alpha}$ is clear from its definition, we can conclude that $\overline{\mathbb{B}}_{\alpha}$ indeed satisfies the axioms of an $L$-fuzzy bornology on the $L$-valued set $\left(X, E_{\alpha}\right)$.

Proposition 7.7. Let the families $\mathbb{B}_{\alpha}$, and $\overline{\mathbb{B}}_{\alpha}: \alpha \in M$ be defined as above. Then:

(5L2B) $\alpha \leq \beta \Rightarrow \mathbb{B}_{\alpha} \geq \mathbb{B}_{\beta} ; \alpha \leq \beta \Rightarrow \overline{\mathbb{B}}_{\alpha} \geq \overline{\mathbb{B}}_{\beta}$;

$(6 \mathrm{~L} 2 \mathrm{~B}) \overline{\mathbb{B}}_{1_{M}}=\mathbb{B}_{1_{M}}=\mathbb{B}$, that is $\mathbb{B}_{1_{M}}$ is the original bornology;

(7L2B) $\overline{\mathbb{B}}_{0_{M}}=L^{X}$, that is $\overline{\mathbb{B}}_{0_{M}}$ is the whole L-power-set of the set $X$.

Indeed, property $(5 \mathrm{~L} 2 \mathrm{~B})$ is clear from the definition of $\mathbb{B}^{\alpha}$, property (6L2B) is clear from property $(8 \mathrm{e})$ of the operator $e^{1_{M}}$ and to establish property $(7 \mathrm{~L} 2 \mathrm{~B})$ we refer to the property $(7 \mathrm{e})$ of the operator $e^{0_{M}}$ and apply axiom $(1 \mathrm{LB})$ of the original $L$-bornology $\mathbb{B}$.

Thus we have obtained a non-increasing family of $L$-fuzzy bornology bases $\left\{\mathbb{B}_{\alpha} \mid \alpha \in M\right\}$ and a family of $L$-fuzzy bornologies $\left\{\overline{\mathbb{B}}_{\alpha} \mid \alpha \in M\right\}$ on the family of $L$-valued sets $\left(X, E_{\alpha}\right)$. We use this family to construct an $M$-valued bornology $\mathcal{B}: L^{X} \rightarrow M$ on the set $X$ by setting

$$
\mathcal{B}(A)=\bigvee\left\{\alpha \in M^{\circ} \mid A \in \overline{\mathbb{B}}_{\alpha}\right\} .
$$

THEOREM 7.8. The mapping $\mathcal{B}: L^{X} \rightarrow M$ constructed above is an $M$ valued bornology on the L-powerset of the set $X$

Proof. The validity of axiom (1LMB) follows from the property $(1 L B)$ which holds for every $L$-bornology $\mathbb{B}_{\alpha} \cdot{ }^{4}$

To verify $(2 \mathrm{LMB})$ notice that from the construction it is clear that if $A \leq B$ and $B \in \mathbb{B}_{\alpha}$ for some $\alpha \in M$, then $A \in \mathbb{B}_{\alpha}$ and hence $\mathcal{B}(A) \leq \mathcal{B}(B)$.

To verify (3LMB) assume that $P, Q \in L^{X}$ and $\mathcal{B}(P) \wedge \mathcal{B}(Q) \geq \alpha$. Then for every $\beta<\alpha$ we have $P \in \overline{\mathbb{B}}_{\beta}, Q \in \overline{\mathbb{B}}_{\beta}$ and hence $P \wedge Q \in \mathbb{B}_{\beta}$. Since this holds for every $\beta<\alpha$ we conclude that $\mathcal{B}(P \wedge Q) \geq \alpha$ and hence $\mathcal{B}(P \wedge Q) \geq$ $\mathcal{B}(P) \wedge \mathcal{B}(Q)$.

REMARK 7.9. The $L$-valued sets $\left(X, E^{\alpha}\right)$ were used when constructing $\alpha$-level $L$-fuzzy bornologies. However, their role is hiden in the constructed $L M$-valued bornology $\mathcal{B}: L^{X} \rightarrow M$ since we view it on the $L$-powerset $L^{X}$

\footnotetext{
${ }^{4}$ Here we refer to $\mathbb{B}^{\alpha}$ as an $L$-bornology and omit the adjective fuzzy, since we deal with $X$ as a set and not as an $L$-valued set $\left(X, E^{\alpha}\right)$
} 
of the crisp set $\mathrm{X}$, that is the $L$-powerset of a set with the ordinary equality $(X,=)$. The way how $\mathcal{B}$ was constructed and the properties of the operator of extensinal hull $e: L^{X} \rightarrow L^{X}$ established in Proposition 7.4 makes it natural to conjecture the following property of constructed $L M$-valued bornology $\mathcal{B}$ :

$$
\mathcal{B}(A) \geq \alpha \Longrightarrow \mathcal{B}\left(e^{\alpha}(A)\right) \geq \alpha \forall \alpha \in[0,1], \forall A \in L^{X} .
$$

Unfortunately, we could not establish this property of the constructed $L M$ valued bornology $\mathcal{B}$.

\section{Conclusion}

We have introduced the concept of an $L M$-valued bornology, that is an $M$-valued bornology on the $L$-powerset $L^{X}$ of an $L$-valued set $(X, E)$. We consider this concept to be an appropriate framework for the study of bornological-type structures in the context of many-valued, in particular fuzzy, mathematical structures. The research of $L M$-bornological spaces and the appropriately defined category of such spaces as objects and bounded fuzzy functions is initiated. A scheme that allows to construct $L M$-valued bornologies, in particular $L M$-bornologies with prescribed properties is presented. This scheme is based on the use of a continuous family of many-valued equalities on the metric space $X$.

As the main directions where the further research should be carried out we view the following.

To conduct a deeper research of the intrinsic properties of the categories $\mathcal{F B O R N}(L, M), \mathcal{F} \mathcal{S B O R N}(L, M)$ and its important subcategories as well as to study the relations of these categories with some other "related" categories, in particular, with categories of fuzzy topology.

To find other substantial examples of $L M$-valued bornological spaces. The main example of $L M$-valued bornologies considered in this paper is based on the use of metrics. We assume it to be interesting and useful to consider other types of $L M$-valued bornologies, in particular many-valued versions of the bornology of relatively compact sets in a topological space and of the bornology of totally bounded subsets of a uniform space.

Taking into account functional analysis as the main source of the concept of bornology, we assume it to be important to consider $L M$-valued bornologies on topological linear spaces or on topological algebras, either fuzzy or crisp.

ACKNOWLEDGEMENTs.

The authors are thankful to the anonymous referee for careful reading the paper and making some useful comments. 


\section{REFERENCES}

[1] M. Abel and A. Šstak, Towards the theory of L-bornological spaces, Iran. J. Fuzzy Syst. 8 (2011) 19-28.

[2] G. Beer, S. Naimpally and J. Rodríguez-López, S-topologies and bounded convergences, J. Math. Anal. Appl. 339 (2008), 542-552.

[3] G. Beer and S. Levi, Gap, excess and bornological convergence, Set-Valued Anal. 16 (2008), 489-506.

[4] G. Beer and S. Levi, Strong uniform continuity, J. Math. Anal. Appl. 350 (2009) 568-589.

[5] G. Beer and S. Levi, Total boundedness and bornology, Topology Appl. 156 (2009) $1271-1288$.

[6] U. Bodenhofer, Ordering of fuzzy sets based on fuzzy orderings. I: The basic approach. Mathware Soft Comput. 15 (2008) 201-218.

[7] C. L. Chang, Fuzzy topological spaces, J. Math. Anal. Appl. 24 (1968), 182-190.

[8] M. Demirci, Fuzzy functions and their fundamental properties, Fuzzy Sets and Systems 106 (1999), 239-246.

[9] M. Demirci, Fundamentals of $M$-vague algebra and $M$-vague arithmetic operations, Internat. J. Uncertain. Fuzziness Knowledge-Based Systems 10 (2002), 25-75.

[10] R. Engelking, General Topology, Panstwowe Wydawnictwo Naukowe, Warszawa, 1977.

[11] G. Gierz, K. H. Hoffman, K. Keimel, J. D. Lawson, M. W. Mislove and D. S. Scott, Continuous Lattices and Domains, Cambridge University Press, Cambridge, 2003.

[12] J. A. Goguen, L-fuzzy sets, J. Math. Anal. Appl. 18 (1967), 145-174.

[13] J. A. Goguen, The fuzzy Tychonoff theorem, J. Math. Anal. Appl. 43 (1973), 734-742.

[14] H. Hogbe-Nlend, Bornology and Functional Analysis, Math. Studies 26, NorthHolland, Amsterdam, 1977.

[15] U. Höhle, Upper semicontinuous fuzzy sets and applications, J. Math. Anal. Appl. 78 (1980), 659-673.

[16] U. Höhle, M-valued sets and sheaves over integral commutative cl-monoids, Chapter 2 in: Applications of Category Theory to Fuzzy Sets, S. E. Rodabaugh, E. P. Klement and U. Höhle (Eds.), Kluwer Acad. Publ., Dordrecht, 1992, pp. 33-72.

[17] U. Höhle, Commutative, residuated l-monoids, in: S. E. Rodabaugh, E. P. Klement and U. Höhle (Eds.), Non-classical logics and their applications to Fuzzy Sets, Kluwer Acad. Publ., Dordrecht, Boston, 1995, pp. 53-106.

[18] U. Höhle, Many-valued equalities, singletons and fuzzy partitions, Soft computing 2 (1998), 134-140.

[19] U. Höhle, Many Valued Topology and its Application, Kluwer Acad. Publ., Boston, Dordrecht, London, 2001.

[20] U. Höhle, H.-E. Porst and A. Šostak, Fuzzy functions: a fuzzy extension of the category SET and some related categories, Appl. Gen. Topol. 1 (2000), 115-127.

[21] S.-T. Hu, Boundedness in a topological space, J. Math. Pures Appl. 28 (1949), 287-320.

[22] S.-T. Hu, Introduction to General Topology, Holden-Day, San Francisco, 1966.

[23] F. Klawonn, Fuzzy points, fuzzy relations and fuzzy functions, in: V. Novák, I. Perfilieva (Eds.), Discovering the World with Fuzzy Logic, Physica, Heidelberg, 2000, pp. 431-453.

[24] E. P. Klement, R. Mesiar, E. Pap, Triangular Norms, Kluwer Acad. Publ., Dordrecht, 2000.

[25] T. Kubiak, On Fuzzy Topologies, PhD Thesis, Adam Mickiewicz University, Poznań, Poland, 1985.

[26] A. Lechicki, S. Levi and A. Spakowski, Bornological convergence, J. Math. Anal. Appl. 297 (2004), 751-770. 
[27] Y.-M. Liu and M.-K. Luo, Fuzzy Topology, Advances in Fuzzy Systems - Applications and Topology, World Scientific, Singapore, New Jersey, London, Hong Kong, 1997.

[28] W. Morgan, and R. P. Dilworth, Residuated lattices, Trans. Amer. Math. Soc. 45 (1939) 335-354. Reprinted in: K.Bogart, R. Freese and J. Kung (Eds.), The Dilworth Theorems: Selected Papers of R. P. Dilworth, Birkhäuser, Basel, 1990.

[29] S. Osçağ, Bornologies and bitopological function spaces, Filomat 27 (2013), 1345-1349.

[30] J. Paseka, S. A. Solovyov and M. Stehlik, Lattice-valued bornological systems, Fuzzy Sets and Systems 259 (2015), 68-88.

[31] I. Perfilieva, Fuzzy function: Theoretical and practical point of view, in: Proc. 7th Conf. European Society for Fuzzy Logic and Technology, EUSFLAT 2011, Aix-LesBains, France, July 18-22, 2011, Atlantis Press, 2011, pp. 480-486.

[32] I. Perfilieva and A. Šostak, Fuzzy functions: Basics of the Theory and Applications to Topology, preprint, 2015.

[33] G. N. Raney, A subdirect-union representation for completely distibutive complete lattices, Proc. Amer. Math. Soc. 4 (1953), 518-522.

[34] S. E. Rodabaugh, Powerset operator based foundation for point-set lattice-theoretic (POSLAT) fuzzy set theories and topologies, Quaestiones Math. 20 (1997), 463-530.

[35] K. I. Rosenthal, Quantales and Their Applications, Pirman Research Notes in Mathematics 234, Longman Scientific \& Technical, 1990.

[36] H. H. Schaefer, Topological Vector Spaces, Springer-Verlag, 1970

[37] A. Šostak, On a fuzzy topological structure, Suppl. Rend. Circ. Mat. Palermo Ser II 11 (1985), 89-103.

[38] A. Šostak, Two decades of fuzzy topology: Basic ideas, notions and results, Russian Math. Surveys 44 (1989), 125-186.

[39] A. Šostak, Basic structures of fuzzy topology, J. Math. Sci. 78 (1996), 662-701.

[40] A. Šstak, Fuzzy functions and an extension of the category L-TOP of Chang-Goguen $L$-topological spaces, in: Proceedings of the $9^{\text {th }}$ Prague Topological Symposium (2001), Topol. Atlas, North Bay, ON, 2002, pp. 271-294.

[41] A. Šostak and I. Uljane, Bornological structures in the context of L-fuzzy sets, in: 8th Conference of the European Society for Fuzzy Logic and Technology (EUSFLAT 2013), Atlantis Premium Proceedings, pp. 481-488.

[42] A. Šostak and I. Uljane, $L$ - valued bornologies on powersets, Fuzzy Sets and Systems 294 (2016), 93-104.

[43] A. Šostak and I. Uljane, LM -fuzzy bornologies on many valued sets, in: Proceedings of V Congress Turkic World Mathematicians (Kyrgyzstan, Bulan-Sogottu, 5-7 June, 2014), A. Borubajev (Ed.), Bishkek, Kyrgyz Mathematical Society, 2014, pp. 32-40.

[44] L. Valverde, On the structure of F-indistinguishibility operators, Fuzzy Sets and Systems 17 (1985), 313-328.

[45] M. Ying, A new approach to fuzzy topology I, Fuzzy Sets and Systems 39 (1991), 303-321.

[46] L. Zadeh, Fuzzy sets, Information and Control 8 (1965), 338-353.

[47] L. Zadeh Similarity relations and fuzzy orderings, Information Sci. 3 (1971), 177-200.

[48] L. A Zadeh, The concept of a linguistic variable and its application to approximate reasoning I, II, III, Information Sci. 8-9 (1975), 199-257, 301-357, 43-80. 


\section{Bornološke strukture na višeznačnim skupovima}

\section{Alexander Šostak i Ingrīda Uljane}

SAŽETAK. Uvodimo pristup konceptu bornologije u kontekstu višeznačnih matematičkih struktura i razvijamo osnove teorije višeznačnih bornoloških prostora te iniciramo proučavanje kategorije višeznačnih bornoloških prostora i prikladno definiranih omeđenih "preslikavanja" takvih prostora. Napravljena je shema za konstrukciju višeznačnih bornologija s unaprijed zadanim svojstvima. Posebno, ta shema dopušta da se uobičajena bornologija metričkog prostora proširi do višeznačne bornologije na njemu.

Alexander Šostak

Department of Mathematics

University of Latvia

LV-1002 Riga, Latvia

and Institute of Mathematics and Computer Sciences UL

LV-1459 Riga, Latvia

E-mail: aleksandrs.sostaks@lu.lv, aleksandrs.sostaks@lumii.lv

Ingrīda Uljane

Department of Mathematics

University of Latvia

LV-1002 Riga, Latvia

and Institute of Mathematics and Computer Sciences UL

LV-1459 Riga, Latvia

E-mail: ingrida.uljane@lu.lv, ingrida.uljane@lumii.lv

Received: 25.4.2017.

Revised: 21.8.2017. 